\title{
IRE1a regulates skeletal muscle regeneration through myostatin mRNA decay
}

\author{
Shengqi He, ${ }^{1}$ Tingting Fu, ${ }^{2}$ Yue Yu, ${ }^{3}$ Qinhao Liang, ${ }^{1}$ Luyao Li, ${ }^{1}$ ling Liu, ${ }^{2}$ Xuan Zhang, ${ }^{1}$ Qian Zhou, ${ }^{2}$ Qiqi Guo, ${ }^{2}$ Dengqiu Xu, ${ }^{2}$ \\ Yong Chen, ${ }^{1}$ Xiaolong Wang, ${ }^{4}$ Yulin Chen, ${ }^{4}$ Jianmiao Liu, ${ }^{5}$ Zhenji Gan, ${ }^{2}$ and Yong Liu
}

\begin{abstract}
'Hubei Key Laboratory of Cell Homeostasis, College of Life Sciences, The Institute for Advanced Studies, Frontier Science Center for Immunology and Metabolism, Wuhan University, Wuhan, China. ${ }^{2}$ State Key Laboratory of Pharmaceutical Biotechnology and MOE Key Laboratory of Model Animals for Disease Study, Department of Spine Surgery, Nanjing Drum Tower Hospital, The Affiliated Hospital of Nanjing University Medical School, Jiangsu Key Laboratory of Molecular Medicine, Chemistry and Biomedicine Innovation Center (ChemBIC), Model Animal Research Center, Nanjing University Medical School, Nanjing University, Nanjing, China. ${ }^{3}$ Division of Ophthalmology, Beth Israel Deaconess Medical Center, Boston, Massachusetts, USA. ${ }^{4}$ Key Laboratory of Animal Cenetics, Breeding and Reproduction of Shaanxi Province, College of Animal Science and Technology, Northwest A\&F University, Yangling, China. ${ }^{5}$ Cellular Signaling Laboratory, Key Laboratory of Molecular Biophysics of Ministry of Education, Huazhong University of Science and Technology, Wuhan, China.
\end{abstract}

\begin{abstract}
Skeletal muscle can undergo a regenerative process in response to injury or disease to preserve muscle mass and function, which are critically influenced by cellular stress responses. Inositol-requiring enzyme 1 (IRE1) is an ancient endoplasmic reticulum stress sensor and mediates a key branch of the unfolded protein response. In mammals, IRE1 $\alpha$ is implicated in the homeostatic control of stress responses during tissue injury and regeneration. Here, we show that IRE1 $\alpha$ serves as a myogenic regulator in skeletal muscle regeneration in response to injury and muscular dystrophy. We found in mice that IRE1 $\alpha$ was activated during injury-induced muscle regeneration, and muscle-specific IRE1 $\alpha$ ablation resulted in impaired regeneration upon cardiotoxin-induced injury. Gain- and loss-of-function studies in myocytes demonstrated that IRE1 $\alpha$ acts to sustain both differentiation in myoblasts and hypertrophy in myotubes through regulated IRE1-dependent decay (RIDD) of mRNA encoding myostatin, a key negative regulator of muscle repair and growth. Furthermore, in the mouse model of Duchenne muscular dystrophy, loss of muscle IRE1 $\alpha$ resulted in augmented myostatin signaling and exacerbated the dystrophic phenotypes. These results reveal a pivotal role for the RIDD output of IRE1 $\alpha$ in muscle regeneration, offering insight into potential therapeutic strategies for muscle loss diseases.
\end{abstract}

\section{Introduction}

The endoplasmic reticulum (ER) is an essential membrane network that governs the folding and secretion of nearly one-third of the cellular proteome in eukaryotes. Increased protein folding demand or accumulation of unfolded/misfolded proteins within the ER lumen causes ER stress, activating the adaptive cell response termed the unfolded protein response (UPR) (1-3). Among the 3 ER-resident transmembrane UPR sensors (3), inositolrequiring enzyme 1 (IRE1) is the most conserved signal transducer, with dual enzyme activities, i.e., Ser/Thr protein kinase and endoribonuclease (RNase), within its cytoplasmic portion $(1,2,4)$. Upon ER stress, IRE1 is activated through dimerization/oligomerization and autophosphorylation, mediating a key branch of the UPR signaling through its RNase activity $(1-3,5,6)$. Activated IRE1 catalyzes the unconventional splicing of the mRNA encoding the transcription factor X-box binding protein 1 (XBP1) to generate a spliced active form of XBP1 (XBP1s), thereby driving a major UPR program involved in modulating protein folding, secretion, and ER-associated degradation $(5,6)$. In addition, IRE1 can also directly

Conflict of interest: The authors have declared that no conflict of interest exists. Copyright: @ 2021, American Society for Clinical Investigation.

Submitted: August 31, 2020; Accepted: July 14, 2021; Published: September 1, 2021.

Reference information: J Clin Invest. 2021;131(17):e143737.

https://doi.org/10.1172/JCl143737. degrade select mRNA species in a process referred to as "regulated IRE1-dependent decay" (RIDD) (7-9). In mammals, IRE1 $\alpha$ signaling has been implicated in regulating a wide range of biological processes, including determination of cell fate, metabolic homeostasis, and immune responses (10-14). Whereas XBP1s serves as a crucial effector of IRE1 $\alpha$ 's RNase activation, recent studies have also revealed implications of its RIDD activity in the control of secretory cargo proteins $(8,15)$, insulin production in pancreatic $\beta$ cells (16), hepatic lipid metabolism (17), and dendritic cell homeostasis in immunity (18). Moreover, a growing body of evidence suggests that dysregulated activation of IRE1 $\alpha$ contributes to the pathogenesis of many chronic diseases, including metabolic disorders, cancer, and neurodegeneration (1,11, 19-22).

Skeletal muscles have the remarkable capacity to undergo regenerative growth in response to injury or other external stimuli. Muscle repair is a highly coordinated process involving muscle damage, regeneration, and myofiber remodeling (23-25). Muscle regeneration relies on activation and expansion of myogenic stem cells, known as satellite cells, residing beneath the basal lamina of myofibers, followed by myoblast differentiation and ultimate fusion into multinucleated myotubes (26-28). At the final stage of muscle repair, myotubes undergo hypertrophy remodeling to generate mature muscle fibers and restore their contractile capacity. Multiple myogenic signaling pathways serve to control muscle regeneration and hypertrophy (27-29), 
mainly mediated by orchestrated activation of the transcriptional regulatory factors MYF5, MyoD, and myogenin, along with regulated expression of the myosin heavy chain (MyHC) genes $(27,28)$. In addition, a number of cytokines and growth factors are known to be critically involved in controlling muscle repair and growth, as exemplified by IL- 6 , myostatin/TGF- $\beta$, and insulin-like growth factor-1 (IGF-1) $(30,31)$. Impairment of muscle regeneration and growth leads to muscle atrophy or dystrophy, which represents a key pathological feature of many human diseases with muscle loss (32). For instance, Duchenne muscular dystrophy (DMD), caused by mutations in the X-linked gene dystrophin, is the most common and severe form of human muscular dystrophy and remains incurable to date $(33,34)$. A better understanding of the complex interplays between the intrinsic cell signaling network and microenvironmental cues will uncover the molecular regulatory mechanisms in the maintenance of muscle mass, thus facilitating the development of regenerative therapeutics for muscle degeneration disorders.

As a critical regulator of ER stress response in restoration of proteostatic homeostasis, IRE1 $\alpha$ has been documented as promoting cell proliferation during the reparative regeneration of the liver (35) and the compensatory growth of pancreatic islets under metabolic stress (36). However, it has yet to be explored whether IRE1 $\alpha$ plays a role in sensing skeletal muscle stress and regulating its regeneration process. In this study, we used a cardiotoxin-induced (CTX-induced) acute muscle injury model as well as the genetic $m d x$ model of DMD in mice, and found that IRE1 $\alpha$ acts to sustain muscle repair and growth, largely through its RIDD activity to downregulate the expression of the mRNA encoding myostatin, a critical negative regulator of muscle regeneration and growth. Our findings demonstrate the physiological importance of IRE1 $\alpha$ 's RIDD activity in the control of muscle regeneration, and its dysregulation may represent an unrecognized mechanism linking ER stress to muscle degenerative diseases.

\section{Results}

Activation of the IRE1 $\alpha$ signaling branch is implicated in skeletal muscle regeneration. To investigate whether IRE1 $\alpha$ is implicated in regulating muscle regeneration, we first examined its activation states during the regenerative response to acute skeletal muscle injury by injecting CTX into the tibialis anterior (TA) muscles of adult mice. In line with previously reported findings (37), CTXinduced myofiber degeneration triggered robust muscle regeneration responses, as indicated by prominently elevated expression of embryonic MyHC (eMyHC) at 2 days after CTX treatment (Figure 1A). Starting at 2 days after CTX-elicited injury, we observed significant increases in the phosphorylation at $\operatorname{Ser}^{724}$ within the kinase domain of IRE1 $\alpha$ that peaked at 4 and 8 days (Figure 1A). Consistent with activated IRE1 $\alpha$ 's RNase activity, significantly increased Xbp1 mRNA splicing and decreased abundance of Blos 1 mRNA, a typical RIDD substrate of IRE1 $\alpha$, were detected during the regenerative response to CTX-induced injury (Figure 1B), in parallel with marked activation of the Myog and Myh8 gene expression program (Figure 1C). Notably, increased eIF2 $\alpha$ phosphorylation and BiP protein expression levels were also seen following CTX treatment (Figure 1A). These results indicate that the IRE1 $\alpha$ branch of the UPR is activated in response to muscle damage, and suggest that IRE1 $\alpha$ may act as a component in the control mechanism during muscle repair and regenerative growth response.

To determine the importance of IRE1 $\alpha$ in skeletal muscle regeneration, we used $E r n 1^{f / f l}$ mice with 2 loxP sites flanking exon 2 of the Ern1 gene (38) and Myod1-Cre mice to generate skeletal muscle-specific IRE1 $\alpha$-knockout (referred to as Ern1 $1^{f / f l}$ Myod1-Cre) mice, which were born at normal Mendelian ratios and appeared to be grossly normal. Immunoblot analysis showed substantial reductions in IRE1 $\alpha$ protein levels from multiple muscle types in Ern1 $1^{f l / f l}$ Myod1-Cre mice relative to their Ern1 $1^{f l f l}$ littermates or Myod1-Cre controls, whereas no changes were detected in their hearts (Supplemental Figure 1A; supplemental material available online with this article; https://doi.org/10.1172/JCI143737DS1). As expected, markedly reduced $X b p 1$ mRNA splicing was detected in the Ern $1^{f / f l}$ Myod1-Cre muscles relative to their control groups (Supplemental Figure 1B). Ern1 ${ }^{f l f l}$ Myod1-Cre mice exhibited no overt differences in their body weight, muscle weight, or myofiber morphology (Supplemental Figure 2, A-D), and showed no significant alterations in the abundance of Atf4 or Chop mRNA (Supplemental Figure 2E) or of $M y h 1, M y h 2$, or Myh4 mRNA (Supplemental Figure 2F) in their TA muscles. This suggests that ablation of IRE1 $\alpha$ neither provokes ER stress, nor affects muscle development. When subjected to CTX-induced injury in their TA muscles, animals of all 3 genotypes showed markedly reduced TA muscle weight, presumably due to extensive myofiber degeneration, at 4 days after CTX injection (Figure 1D). Whereas gradual restoration of TA muscle mass was seen in all 3 genotypes at 8 and 12 days after CTX injection (Figure 1D), a significant reduction in the weight of regenerating TA muscle was observed in Ern $1^{f / f l}$ Myod1-Cre mice relative to their Ern $1^{f / f l}$ or Myod1-Cre counterparts at 12 days after CTX-induced injury (Figure 1D). This indicates that loss of IRE1 $\alpha$ in muscle cells resulted in impaired regenerative response to acute muscle injury. Indeed, histological analysis revealed that at 8 days after CTX injection, while TA muscles from all 3 genotypes showed newly formed myofibers containing centralized nuclei that replaced the damaged myofibers (Figure 1E), a significantly lower percentage of large regenerated myofibers was found in Ern1 $1^{f l / f l}$ Myod-Cre TA muscles than in those of Ern1 $1^{f / f l}$ or Myod1-Cre control mice at 8 or 12 days after CTX-induced injury (Figure 1, E and F, and Supplemental Figure 3A). This defect of reparative regeneration in Ern ${ }^{f l / f l}$ Myod1-Cre TA muscles was further reflected by analysis of muscle sections stained for eMyHC (Figure 1, G and H, and Supplemental Figure 3B). At 8 days after CTX injection, whereas Ern1 $1^{f / f l}$ or Myod1-Cre TA muscles exhibited strongly expressed eMyHC in most myofibers, Ern1 ${ }^{f / f l}$ Myod1-Cre TA muscles showed marked reductions in eMyHC expression in many myofibers, along with eMyHC-positive fibers displaying apparent heterogeneity in size (Figure $1, \mathrm{G}$ and $\mathrm{H}$ ). At 12 days after CTX injection, however, Ern1 $1^{f / f l}$ or Myod1-Cre TA muscles had weaker eMyHC signals and larger myofibers, but Ern1 ${ }^{f / f l}$ Myod1Cre TA muscles displayed accumulation of eMyHC in smaller myofibers, likely reflecting an earlier stage of delayed regeneration (Supplemental Figure 3B). Thus, loss of muscle IRE1 $\alpha$ leads to impaired skeletal muscle regeneration after acute injury.

Skeletal muscle regeneration in response to injury involves highly orchestrated myogenic transcriptional network and cytokine signaling. Interestingly, we detected markedly decreased 
A

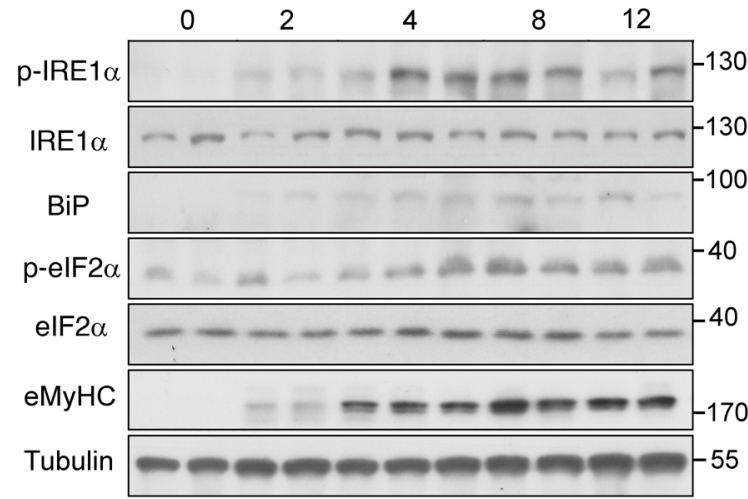

D
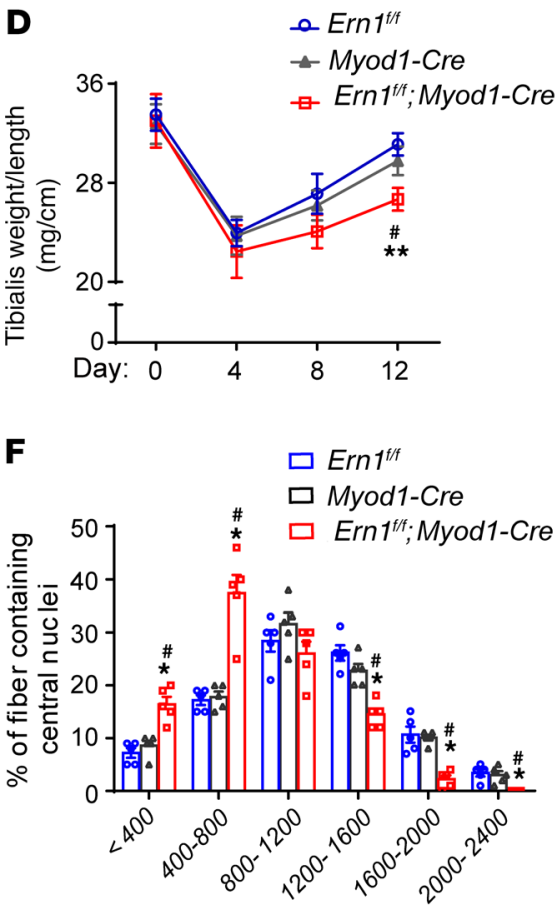

Fiber cross section area $\left(\mu \mathrm{m}^{2}\right)$

G
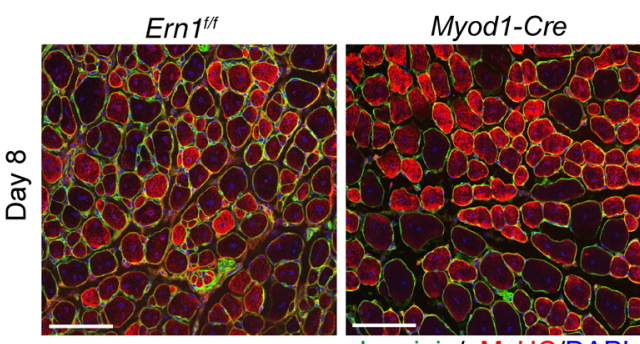

Laminin/eMyHC/DAPI
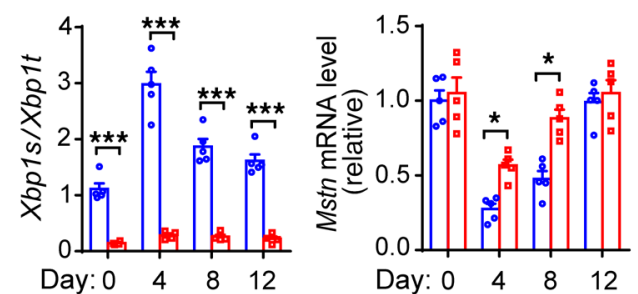

J
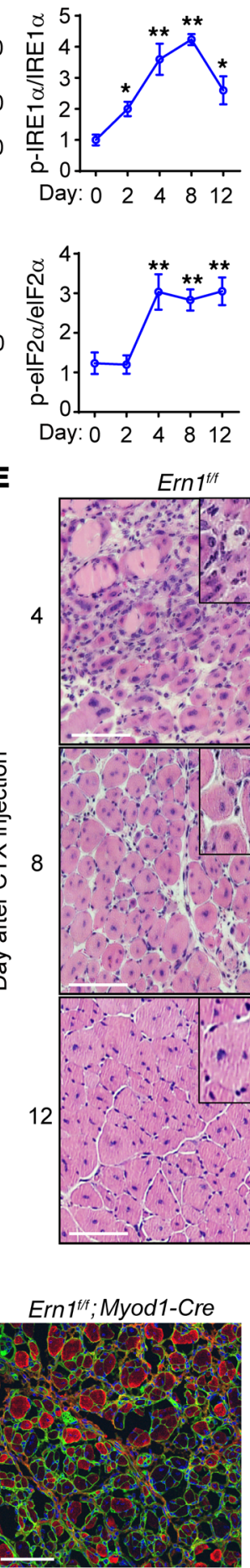

E
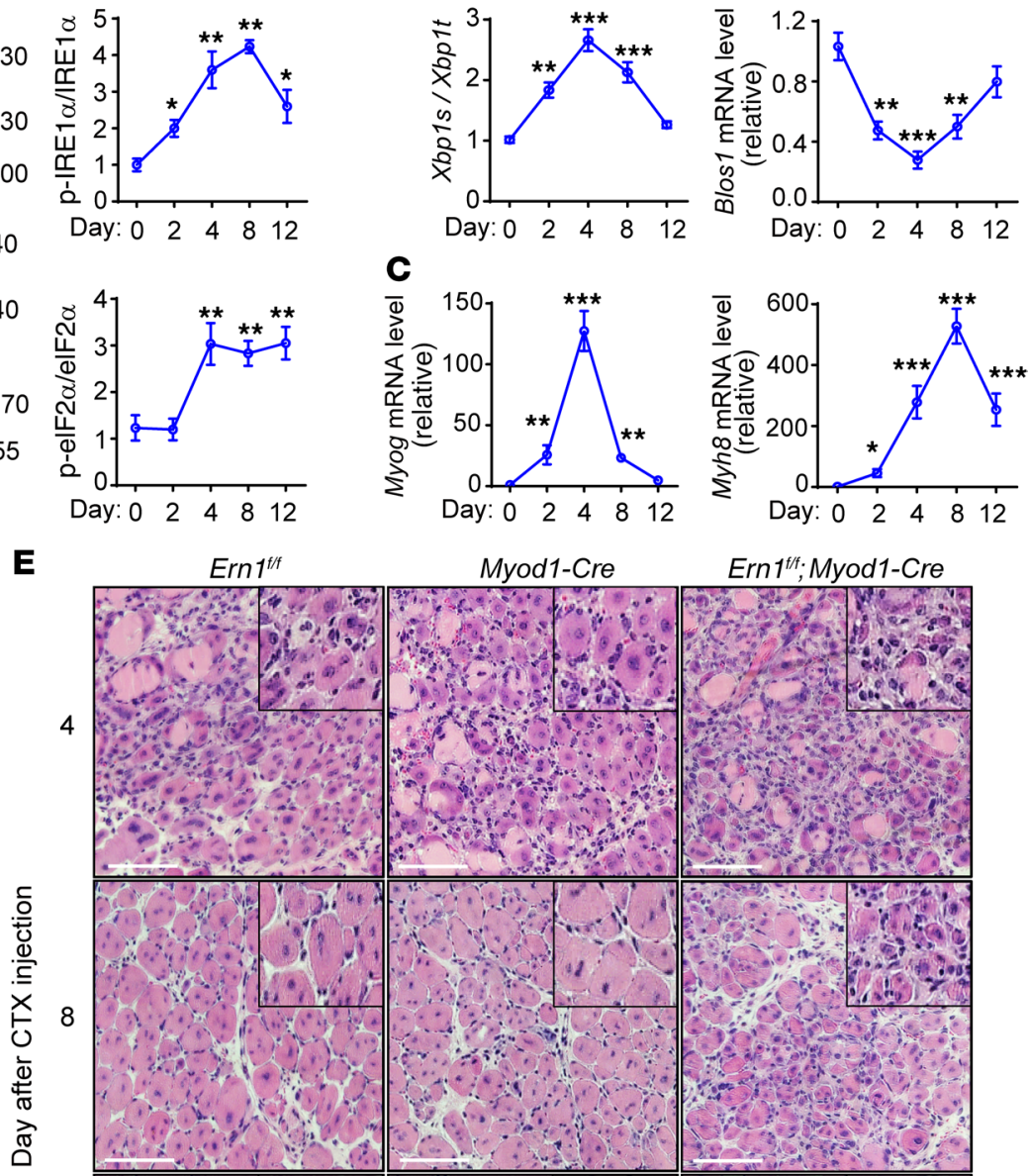

Day: $\begin{array}{lllll}0 & 2 & 4 & 8 & 12\end{array}$

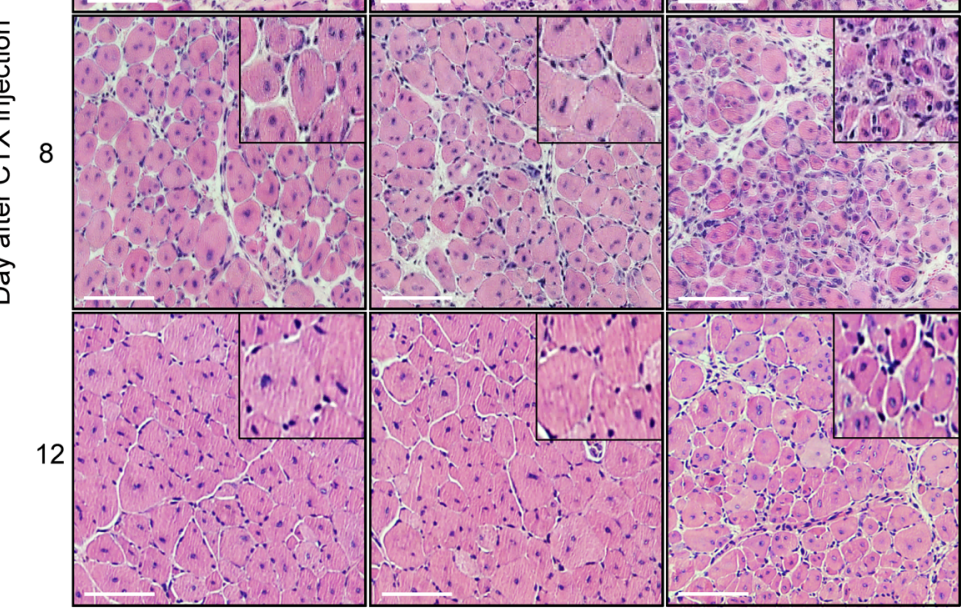

H
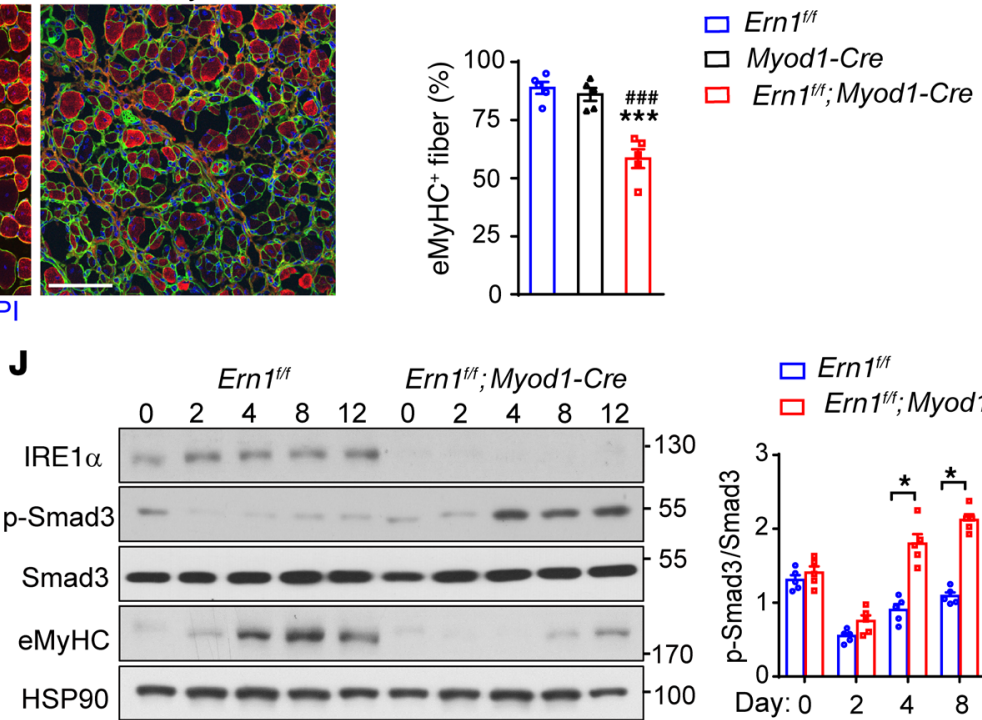

$\square E r n 1^{\text {th }}$

口 Ern1 ${ }^{\text {tf }} ;$ Myod1-Cre

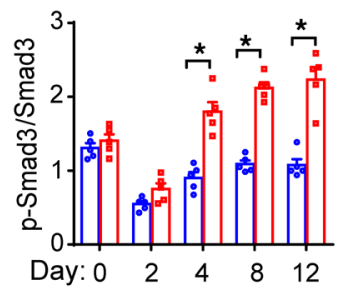


Figure 1. Injury-induced IRE1 $\alpha$ activation affects skeletal muscle regeneration and myostatin signaling. (A-C) Muscle injury induces IRE1 $\alpha$ activation. TA muscle of adult male mice was injected with CTX to induce acute muscle injury ( $n=5$ mice at each time point). (A) Immunoblot analysis of the phosphorylation of IRE $1 \alpha$ and eIF $2 \alpha$, and protein expression of BiP and eMyHC in muscle extracts. Each lane represents 1 individual mouse. Averaged $\mathrm{p}$-IRE1 $\alpha /$ IRE $1 \alpha$ and $\mathrm{p}$-elF2 $\alpha /$ elF2 $\alpha$ ratios are shown from densitometric quantification. (B and C) Quantitative RT-PCR analysis of Xbp1 mRNA splicing and the abundance of mRNAs encoding the indicated genes. (D-J) IRE1 $\alpha$ abrogation impairs muscle regeneration and enhances myostatin signaling. TA muscles of male Ern $7^{f / f l}$ and Myod1-Cre control and Ern $7^{f / f l}$ Myod1-Cre mice were subjected to CTX-induced injuries ( $n=5$ mice at each time point). (D) TA muscle weight relative to tibia length. (E) Representative H\&E staining of TA muscles from mice of the indicated genotypes. (F) Percentage of regenerated myofibers in the indicated cross-sectional areas of TA muscles at 12 days after CTX injection. Myofibers containing centralized nuclei were quantified by ImageJ from 500 myofibers of the TA muscle in each mouse. (G) Representative laminin (green) and eMyHC (red) immunostaining of TA muscles at 8 days after CTX injection. (H) Quantification of the percentage of eMyHC+ myofibers within laminin staining. (I) Quantitative RT-PCR analysis of Xbp1 mRNA splicing and Mstn mRNA abundance in TA muscles. Data are shown as relative to the value for $E r n 7^{f l / f l} \mathrm{TA}$ muscle at day 0 after normalization to Gapdh as the internal control. (J) Immunoblot analysis of TA muscle lysates. Averaged p-Smad/ Smad ratios are shown after normalization to the value for Ern $7^{f / f l} T A$ muscle at day 0 . All data are presented as mean \pm SEM. Significance was calculated by 1-way (A-C and $\mathbf{H}$ ) or 2-way (D, F, I, and J) ANOVA with Bonferroni's multiple-comparison test. ${ }^{*} P<0.05,{ }^{* *} P<0.01$, ${ }^{* * *} P<0.001$ vs. day 0 or Ern $7^{f / f f l}$. $P<<0.05,{ }^{\# \# P}<0.001$ vs. Myod1-Cre. Scale bars: $100 \mu \mathrm{m}$.

Xbp1 mRNA splicing but significantly increased abundance of Mstn mRNA, encoding myostatin (Figure 1I), along with a considerable reduction in Igfl mRNA expression (Supplemental Fig-

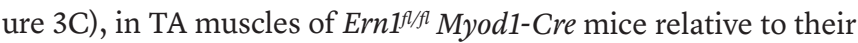
$E r n 1^{f / f l}$ counterparts during the regenerative response at 4 and 8 days after CTX injection. Given that myostatin is a secreted TGF- $\beta$ family member protein that functions as a potent negative regulator of myogenic differentiation and muscle growth (30), this suggests that IRE1 $\alpha$ ablation affected the signaling pathways in the control of muscle regeneration. Indeed, we observed prominently elevated phosphorylation of Smad3, the downstream transcription factor of the myostatin signaling cascade (39), in Ern $1^{\text {fl/l }}$ Myod1Cre TA muscles (Figure 1J). In accordance with poorer muscle regeneration, this enhanced myostatin signaling was associated with marked reductions in eMyHC protein levels (Figure 1J). Together, these results demonstrate that IRE1 $\alpha$ abrogation leads to impaired muscle regeneration and enhanced myostatin signaling in response to acute muscle injury, indicating that IRE1 $\alpha$ may function as a myogenic regulator of muscle repair and growth.

IRE1 $\alpha$ RNase regulates myostatin expression and myoblast differentiation. Because skeletal muscle regeneration involves the activation, proliferation, and differentiation of muscle satellite cells (MuSCs) (28), we first examined whether IRE1 $\alpha$ ablation affected MuSCs in Ern1 ${ }^{f / f l}$ Myod1-Cre mice. Using a FACS-based method (40) for quantitative analysis of either quiescent or activated MuSCs (VCAM1 ${ }^{+}$CD $45^{-}{ }^{-}$CD31-Sca1 ${ }^{-}$cells), we observed no significant differences in the number of MuSCs from CTX-injured TA

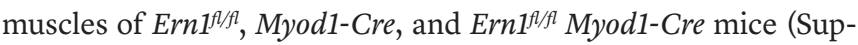
plemental Figure 4, A and B). This suggests that the satellite cell niche was presumably not affected by muscle IRE1 $\alpha$ deficiency.
We then investigated the requirement for IRE1 $\alpha$ in myogenesis

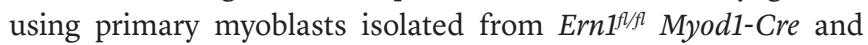
Myod1-Cre mice. During the early period of myoblast differentiation into myotubes, we observed significantly induced IRE1 $\alpha$ phosphorylation (Figure 2, A and B), in parallel with increased Xbp1 mRNA splicing and markedly induced Myh4 expression (Supplemental Figure 5A). Consistently, loss of IRE1 $\alpha$ in primary myoblasts resulted in significantly upregulated expression of Mstn mRNA (Figure 2C), along with elevated Smad3 phosphorylation and reduced MyHC protein level (Figure 2, D and E). At 3 days after differentiation, increased myostatin protein was detected in the culture medium of Ern1-mKO (i.e. Ern1 ${ }^{1 / 1 / 1}$ Myod1-Cre) myotubes (Figure 2F); and most Myod1-Cre myoblasts became elongated and fused with expression of the differentiation marker MyHC (Figure 2, G-I). By contrast, Ern1-mKO myoblasts showed significant decreases in cell fusion and multinuclear myotube formation (Figure 2, G-I), with fewer and shorter myotubes observed after differentiation for 5 days (Supplemental Figure 5B). Moreover, Ern1- $m K O$ myocytes had significant reductions in the mRNA and protein expression levels of the differentiation markers myogenin and MyHC when compared with their Myod1-Cre controls (Figure 2, J-L). These results suggest that IRE1 $\alpha$ ablation leads to upregulation of myostatin expression and impairment of myoblast differentiation in a cell-autonomous manner.

To further test whether IRE1 $\alpha$ 's RNase activity is required for myoblast differentiation, we examined the effects of restored expression of WT or mutant IRE1 $\alpha$ using adenoviruses on the defective differentiation of Ern1-mKO myoblasts. Immunostaining of MyHC showed that overexpression of IRE1 $\alpha$-WT effectively restored the differentiation capacity (Figure 3, A and B) as well as the protein and mRNA expression levels of Myog and Myh4 in Ern1-mKO myoblasts (Figure 3, C-F) to an extent similar to that in Myod1-Cre controls, whereas overexpression of the IRE1 $\alpha$ kinase-dead (KD, K599A) or RNase-dead (RD, K907A) mutant versions (41) lost these effects (Figure 3, A-F). Supporting the role of IRE1 $\alpha$ in suppression of myostatin, overexpression of IRE1 $\alpha$-WT, but not its kinase- or RNasedeficient mutants, substantially blunted the upregulated expression of Mstn in Ern1-mKO myocytes, which resembled the changes of the typical RIDD substrate, Blos1 (Figure 3F). Notably, adenoviral overexpression of XBP1s did not significantly affect the differentiation capacity or Mstn expression in Ern1-mKO myocytes (Figure 3, A-F). These data demonstrate an IRE1 $\alpha$ RNase-dependent, XBP1independent regulation of myoblast differentiation, suggesting that IRE1 $\alpha$ acts as a negative regulator of myostatin to control myoblast differentiation during muscle regeneration.

IRE1 $\alpha$ downregulates myostatin expression and promotes myotube hypertrophy. Because myostatin is also known as an autocrine factor to suppress hypertrophy in myotubes $(30,42,43)$, we next tested whether IRE1 $\alpha$ could impact myotube hypertrophy remodeling by downregulating myostatin expression. In differentiated C2C12 myotubes, adenoviral expression of a short hairpin RNA (shRNA) directed against IRE1 $\alpha$ (Sh-Ern1), in comparison with the scramble shRNA control (Sh-Con), resulted in substantially decreased Xbp1 mRNA splicing, but significantly increased mRNA abundance of Blos1 and Mstn (Figure 4A), accompanied by a significant reduction in the mRNA level of Myh4 but not Igfl (Figure 4A). In line with enhanced myostatin signaling and impaired myotube growth, 
A
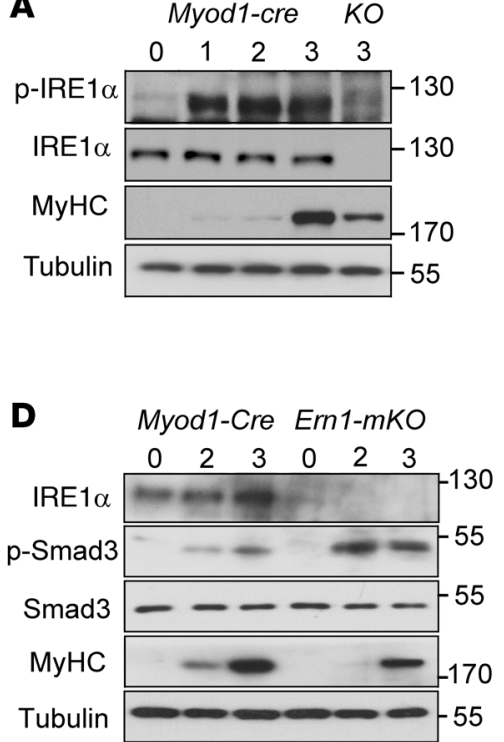

B

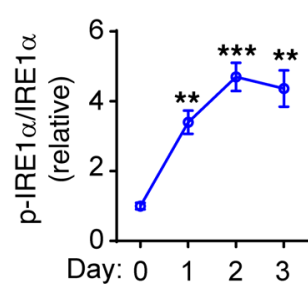

C

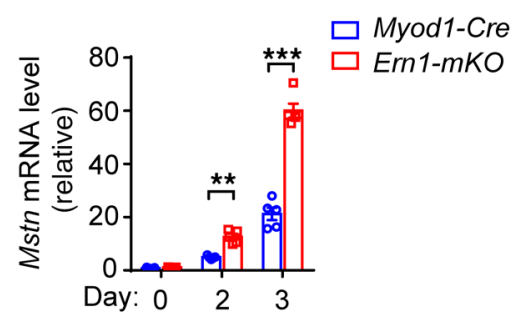

E

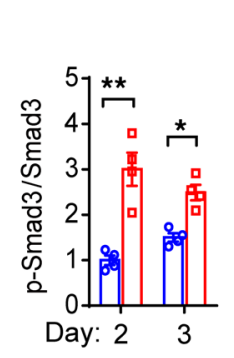

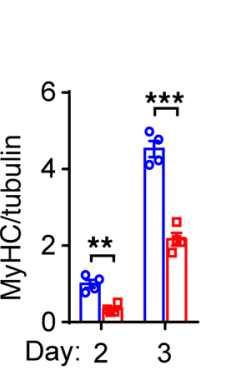

$\mathbf{F}$

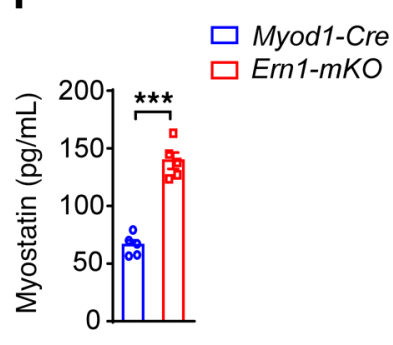

G

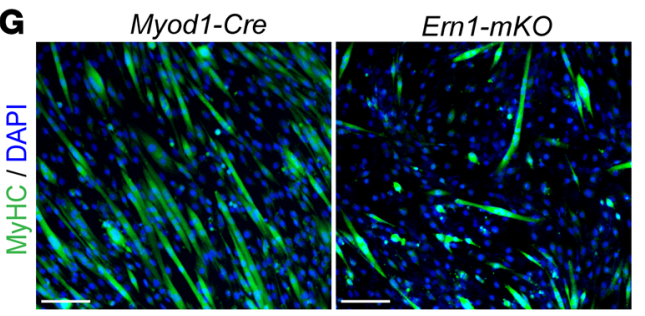

H

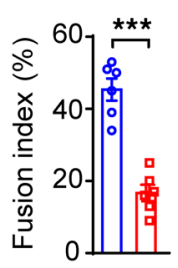

I

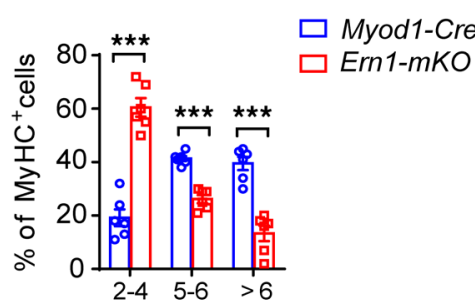

No. of nuclei/fiber
J

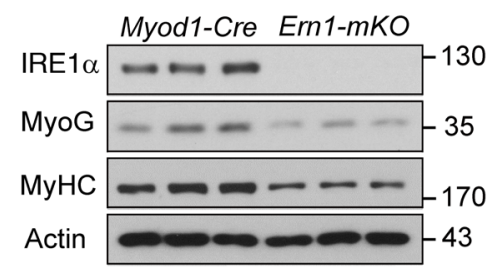

K

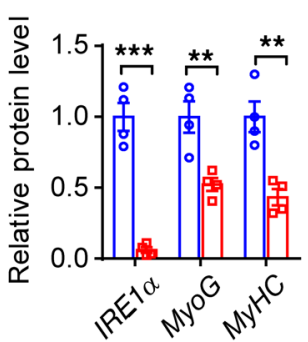

L

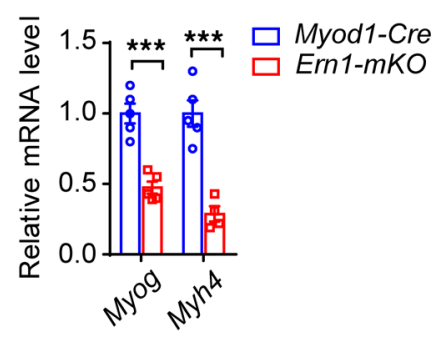

Figure 2. IRE1 $\alpha$ ablation increases myostatin expression and impairs myoblast differentiation. (A-E) Primary myoblasts isolated from TA muscle of male Myod1-Cre and Ern1 ${ }^{f l / f l}$ Myod1-Cre (KO or Ern1-mKO) mice were differentiated for up to 3 days as indicated ( $n=3-5$ independent experiments). (A and B) Immunoblot analysis of IRE1 $\alpha$ phosphorylation and MyHC expression (A) at the indicated time of differentiation, and quantification of averaged p-IRE1 $\alpha /$ IRE1 $\alpha$ ratios (B). (C) Quantitative RT-PCR analysis of the mRNA abundance of Mstn. (D and E) Immunoblot analysis of cell lysates (D) using the indicated antibodies, and averaged p-Smad3/Smad3 and MyHC/tubulin levels (E). (F-L) Primary TA myoblasts were differentiated into myotubes for 3 days ( $n=4-6$ independent experiments). (F) ELISA analysis of myostatin protein in culture medium. (G-I) Representative images (C) of immunostaining of MyHC (green) and DAPI staining of nuclei (blue), with quantification of the fusion index (H) and the number of nuclei per $\mathrm{MyHC}^{+}$fiber (I). (J-L) Immunoblot analysis (J) and quantification (K) of MyoG and MyHC in differentiated myocytes, and quantitative RT-PCR analysis (L) of their mRNA expression levels. All data are presented as mean \pm SEM. Significance was calculated by unpaired 2-tailed Student's $t$ test $(\mathbf{F}, \mathbf{H}, \mathbf{I}$, $\mathbf{K}$, and $\mathbf{L}$ ), 1-way ANOVA (B), or 2-way ANOVA (C and $\mathbf{E}$ ) with Bonferroni's multiple-comparison test. ${ }^{*} P<0.05,{ }^{* *} P<0.01,{ }^{* * *} P<0.001$ vs. Myod1-Cre group, or vs. day 0 for $\mathbf{B}$. Scale bars: $100 \mu \mathrm{m}$.

knockdown of IRE1 $\alpha$ expression led to significantly higher production of myostatin protein in the medium (Figure $4 \mathrm{~B}$ ) and increased phosphorylation of Smad3 (Figure 4C), along with decreases in S6K phosphorylation and $\mathrm{MyHC}$ protein levels. Moreover, this was in parallel with significantly reduced diameters of myotubes (Figure 4D) and total cellular protein contents (Figure 4E). This is con- sistent with the phenotypes of smaller myofibers in mice with muscle IRE1 $\alpha$ abrogation following CTX-induced injury. Conversely, when compared with the control, adenoviral IRE1 $\alpha$ overexpression in differentiated $\mathrm{C} 2 \mathrm{C} 12$ myotubes resulted in significantly elevated Xbp1 mRNA splicing (Figure 4F), reduced mRNA abundance of Blos1 and Mstn (Figure 4F), and lower myostatin protein produc- 

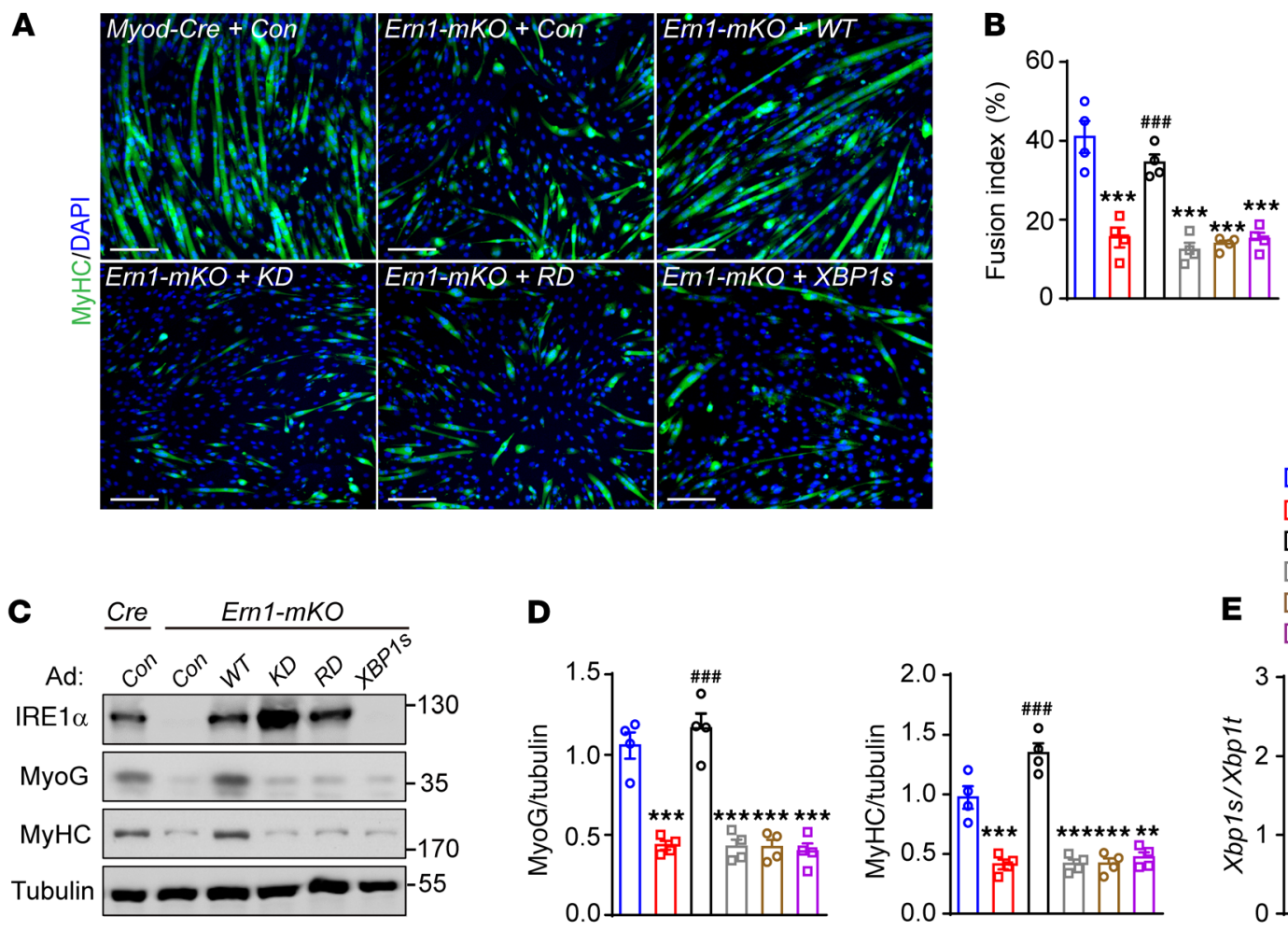

D

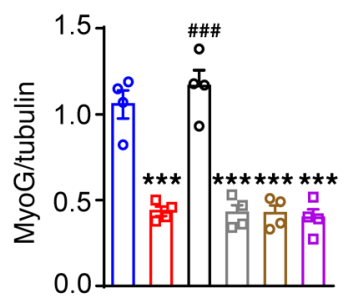

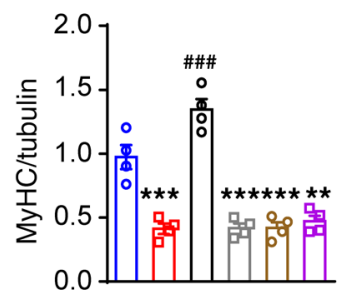
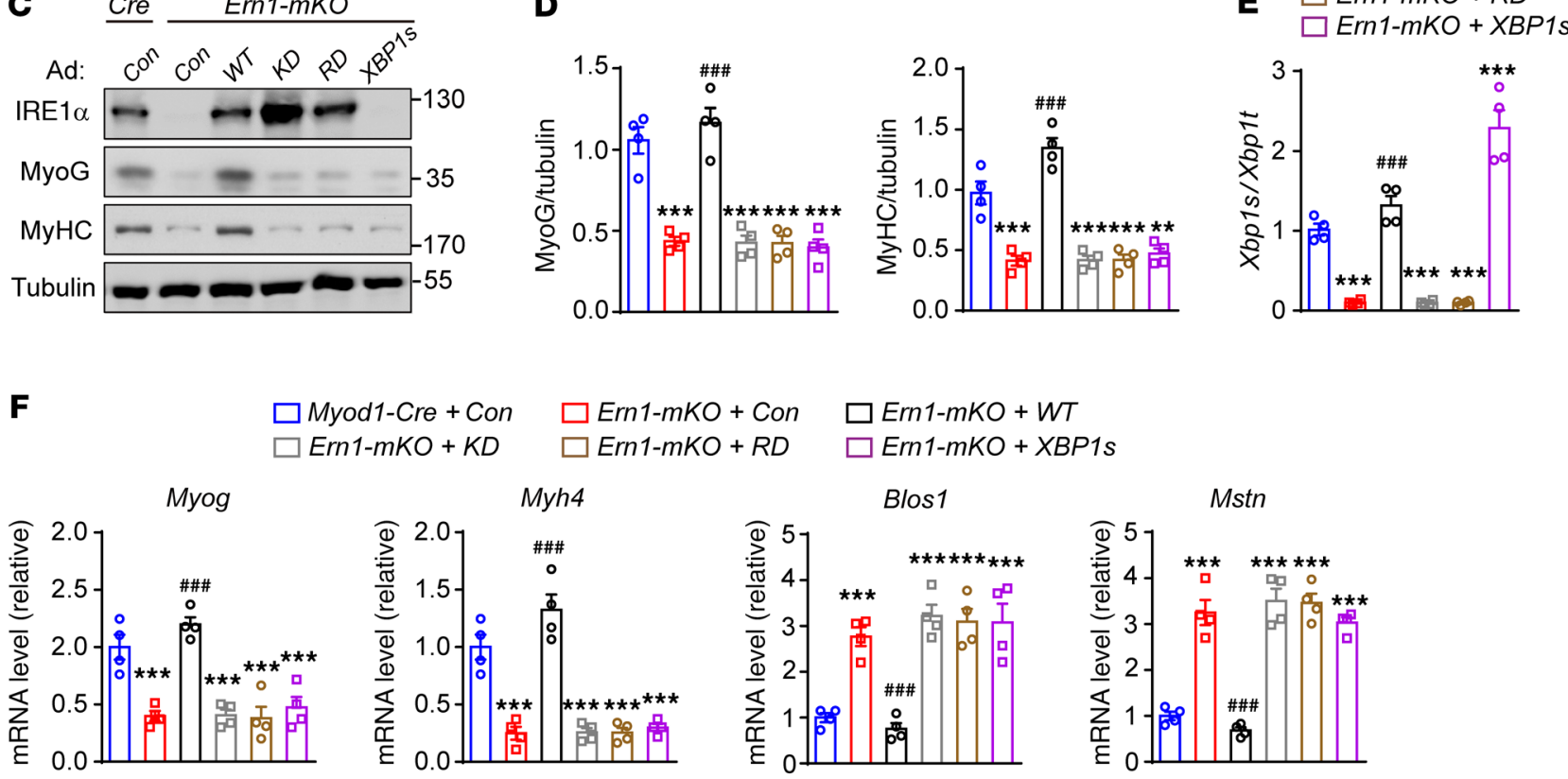

$$
\begin{aligned}
& \text { Myod1-Cre + Con } \\
& \text { Ern1-mKO + KD }
\end{aligned}
$$
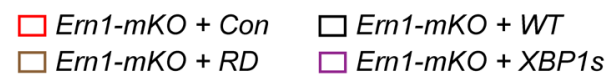

\section{Myh4}
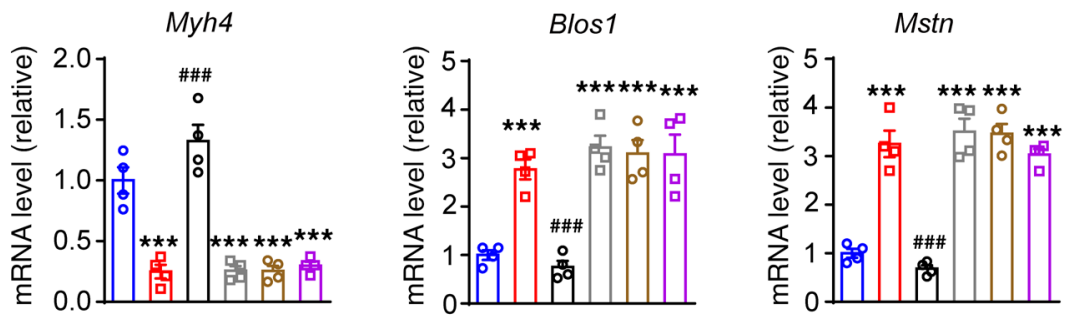

Figure 3. IRE1 $\alpha$ RNase blunts myostatin signaling and promotes myoblast differentiation. Primary Myod1-Cre and Ern1-mKO myoblasts were infected with control recombinant adenovirus or adenoviruses expressing human WT or mutant (KD, kinase dead) IRE1 $\alpha$ protein or XBP1s and then differentiated for 3 days ( $n=4$ independent experiments). (A) Representative images of anti-MyHC and DAPI staining. (B) Quantification of the fusion index. (C) Immunoblot analysis of the indicated proteins. (D) Quantification of MyoG/tubulin and MyHC/tubulin after normalization to the value of control. (E and F) Quantitative RT-PCR analysis of Xbp1 mRNA splicing (E) and the mRNA abundance of the indicated genes (F). All data are presented as mean \pm SEM.

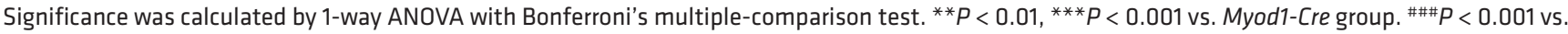
Ern1-mKO + control adenovirus. Scale bars: $100 \mu \mathrm{m}$.

tion (Figure $4 \mathrm{G})$ and signaling (Figure $4 \mathrm{H})$, along with enlarged myotubes (Figure 4I) and higher cellular protein contents (Figure 4J). Notably, adenoviral shRNA knockdown of XBP1 expression caused a slight reduction in Mstn mRNA level (Supplemental Figure 6A), while showing no significant effect on myotube growth (Supplemental Figure 6B). In addition, adenoviral overexpression of IRE1 $\alpha$-WT, but not its KD or RD mutant versions, significantly increased Xbp1 mRNA splicing and decreased the mRNA abundance of Mstn and Blos1 in C2C12 myotubes (Figure 4K). These in vitro analyses via loss- and gain-of-function strategies in myotubes strongly suggest that IRE1 $\alpha$ downregulation of myostatin expression also contributes to the control of myotube hypertrophy remodeling. Since myostatin was reported to impact myoblast pro- liferation (44), we also evaluated whether IRE1 $\alpha$ exerts a regulatory action in this regard. MTT analyses showed that neither deficiency nor overexpression of IRE1 $1 \alpha$ had significant effects on the proliferation rate of primary or $\mathrm{C} 2 \mathrm{C} 12$ myoblasts (Supplemental Figure 7, $\mathrm{A}-\mathrm{C})$. Moreover, FACS analyses showed that knockdown of IRE1 $\alpha$ expression in C2C12 myoblasts did not affect cell death, which was increased upon differentiation stimuli (Supplemental Figure 7D). Thus, IRE1 $\alpha$-directed regulation of myostatin is implicated in both myocyte differentiation and hypertrophy growth, consistent with our observation in vivo that loss of IRE1 1 led to impairment in muscle regeneration upon injury.

Myostatin mediates IRE1a's regulation of muscle cell differentiation and growth. We then determined whether myostatin is required 
A $\square$ Sh-Con

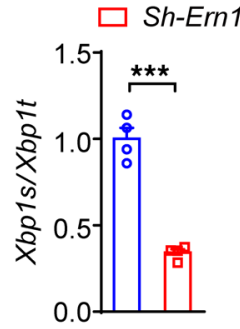

B

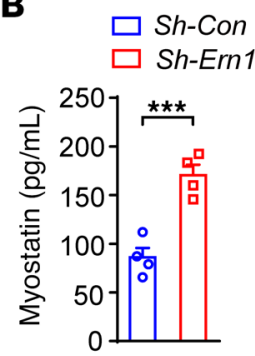

$\mathbf{F}$

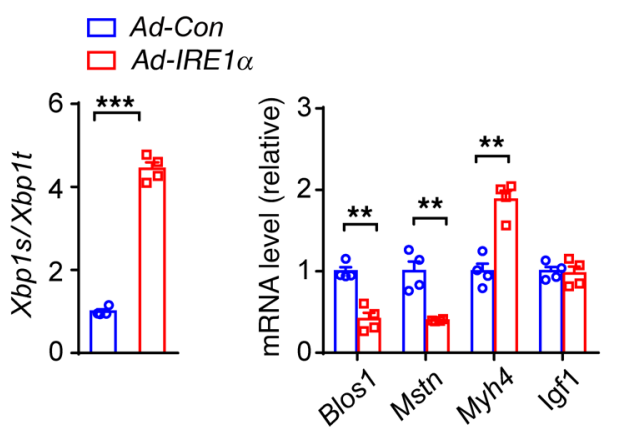

c

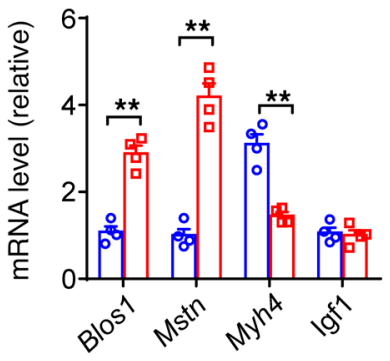

D

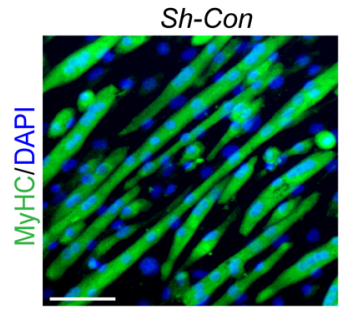

Sh-Ern1

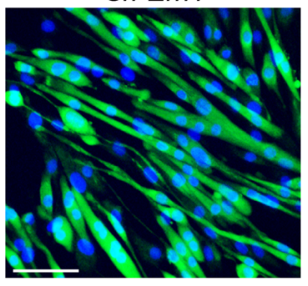

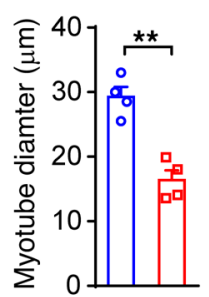

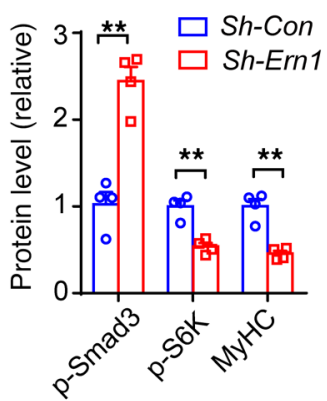

E

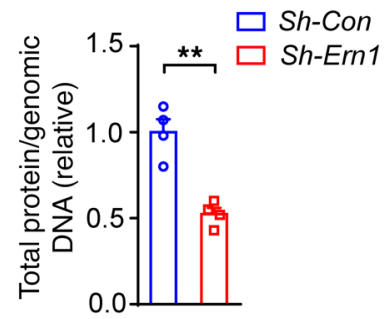

H

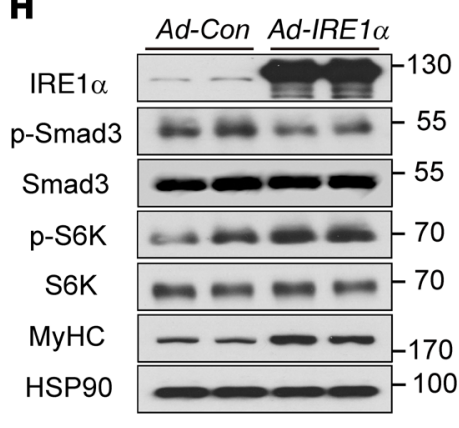

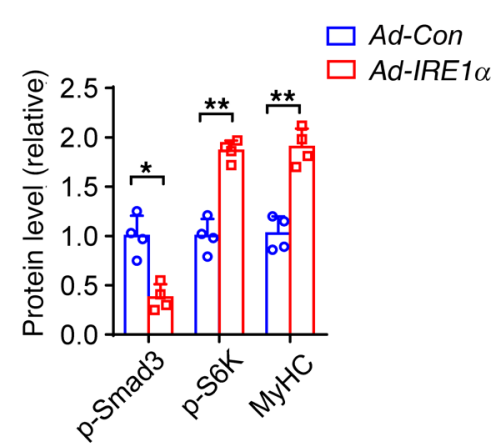

G

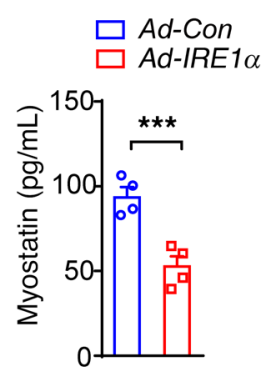

$\mathbf{K}$

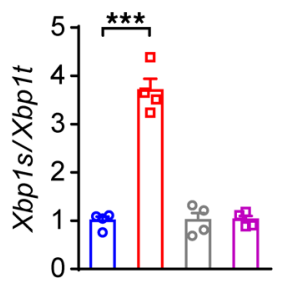

I

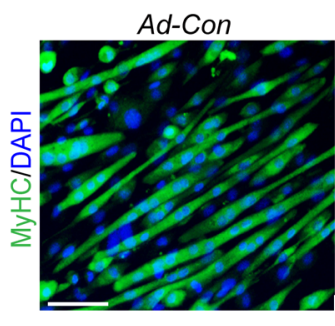

Ad-IRE1a
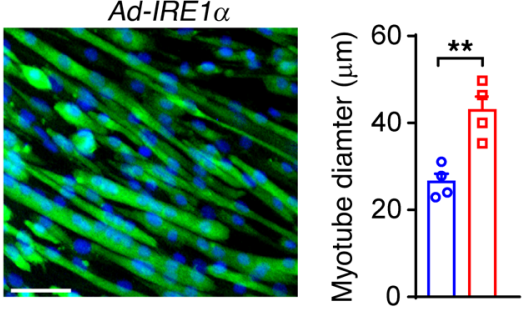

$\mathbf{J}$

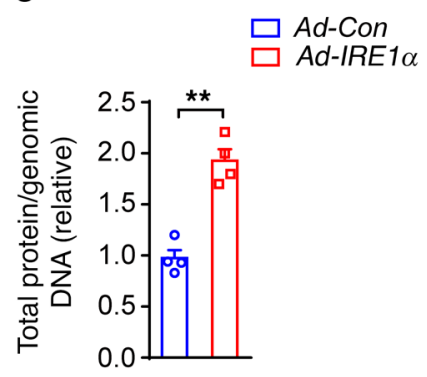


Figure 4. IRE1 $\alpha$ downregulates myostatin expression and promotes hypertrophy of differentiated myotubes. (A-J) C2C12 myoblast cells were differentiated for 4 days into myotubes and then infected for 48 hours with adenoviruses expressing a scramble control shRNA (Sh-Con) or shRNA directed against IRE1 $\alpha$ (Sh-Ern1) (A-E), or with empty control adenovirus (Ad-Con) or that expressing human IRE1 $\alpha$ (Ad-IRE1 $\alpha$ ) (F-J) ( $n$ $=4$ independent experiments). (A and F) Quantitative RT-PCR analysis of Xbp1 mRNA splicing and the mRNA abundance of the indicated genes. (B and $\mathbf{G )}$ ELISA analysis of myostatin protein in culture medium. (C and $\mathbf{H}$ ) Immunoblot analysis of the indicated proteins from myotube extracts. Averaged $\mathrm{MyHC} / \mathrm{actin}, \mathrm{p}-\mathrm{S} 6 \mathrm{~K} / \mathrm{S} 6 \mathrm{~K}$, and $\mathrm{p}-\mathrm{Smad} 3 / \mathrm{Smad} 3$ ratios were normalized to the value of Sh-Con or Ad-Con myotubes. ( $\mathbf{D}$ and I) MyHC immunostaining of myotubes. Myotube diameters were quantified using ImageJ software. (E and J) Total cellular protein content relative to genomic DNA was measured in myotubes. (K) C2C12 myotubes were infected with empty control or adenoviruses expressing the WT or indicated mutant IRE1 $\alpha$ protein. Quantitative RT-PCR analysis of Xbp1 mRNA splicing and the mRNA abundance of Blos1 and Mstn ( $n=4$ independent experiments). All data represent mean \pm SEM. Significance was calculated by unpaired 2-tailed Student's $t$ test (A-J) or 1-way ANOVA (K) with Bonferroni's multiple-comparison test. ${ }^{*} P<0.05,{ }^{* *} P<0.01,{ }^{* *} P<0.001$ vs. Sh-Con or Ad-Con. Scale bars: $100 \mu \mathrm{m}$.

for IRE1 $\alpha$-mediated effect on myoblast differentiation using Mstn-KO C2C12 cells in which Mstn was abrogated by a CRISPR/ Cas9-based strategy (45). Immunostaining of MyHC in differentiated myotubes showed slightly increased fusion index and myotube diameter in Mstn-KO cells relative to the WT control cells (Figure 5, A-C). Whereas knockdown of IRE1 $\alpha$ expression resulted in lower cell fusion and smaller myotube formation in parallel with higher myostatin production (Figure 5D) in WT myoblasts, loss of myostatin diminished these effects of IRE1 $\alpha$ deficiency in MstnKO cells (Figure 5, A-D). Consistently, immunoblotting and quantitative reverse transcription PCR (qRT-PCR) analyses showed that ablation of myostatin restored the protein and mRNA expression of the myogenic markers myogenin and MyHC in the face of IRE1 $\alpha$ knockdown (Figure 5, E-G), with no significant alterations observed in Igfl mRNA level (Figure 5G). Moreover, the promoting effects of IRE1 $\alpha$ overexpression on muscle cell differentiation and growth were also diminished in Mstn-KO cells (Supplemental Figure 8, A-E). These data revealed the importance of myostatin suppression, at least in large part, in mediating IRE1 $\alpha$ regulation of myocyte differentiation and growth. Together, our results support a model in which IRE1 $\alpha$ suppresses myostatin expression, thereby promoting myoblast differentiation and myotube hypertrophy growth during skeletal muscle regeneration.

Myostatin mediates IRE1 $\alpha$ 's regulatory action during skeletal muscle regeneration. To determine the importance of myostatin in mediating IRE1 $\alpha$ 's regulatory effect in vivo on muscle regeneration, we generated the recombinant adeno-associated virus 2/9 (AAV2/9) expressing the N-terminal propeptide (MyoPPT) of myostatin, which is capable of inhibiting the biological activity and function of mature myostatin (46-48). Systemic delivery of MyoPPT $(49,50)$ to neonatal Ern $1^{f / f l}$ and Ern1 $1^{f / f l}$ Myod1-Cre mice, relative to the GFP control, resulted in efficient MyoPPT expression in their TA muscles and did not influence IRE1 $\alpha$ 's Xbp1 mRNA splicing activity (Figure 6, A and B); and in accordance with reported findings $(49,50)$, blocking myostatin signaling by MyoPPT caused significant increases in their body weight and TA muscle weight in both groups (Figure 6, C and D). Importantly, at 8 days after CTX-induced injury, MyoPPT not only promoted the reparative regeneration of TA muscles of the $E r n 1^{f / f l}$ control group, but also prominently rescued the regenerative defect of Ern $1^{f / f l}$ Myod1-Cre TA muscles, as evidenced by significantly increased TA muscle weight (Figure 6E), more newly formed myofibers containing centralized nuclei (Figure 6F), increased eMyHC expression (Figure 6G), and higher percentages of large regenerated myofibers (Figure 6H) and eMyHC-positive fibers (Figure 6I) when compared with the GFP control group (Figure 6, E-I). These results further demonstrate in vivo that myostatin is a crucial player in mediating IRE1 $\alpha$ ablation-associated impairment of skeletal muscle regeneration upon acute injury.

IRE1 1 suppresses myostatin expression through its RIDD activity. We next asked whether the mRNA expression of Mstn is subjected to the control of IRE1 $\alpha$ 's RIDD activity. To determine whether Mstn mRNA represents a valid RIDD substrate, we conducted bioinformatics analysis combined with prediction of mRNA secondary structures. Indeed, we identified a highly conserved region in mammalian mRNAs encoding myostatin (spanning nucleotides 335-349 downstream of the putative transcription start site in mouse Mstn mRNA) that possesses the postulated RIDD consensus sequence (CNGCNN) (9) within a predicted hairpin structure containing a potential IRE1 $\alpha$ cleavage site as found in Xbp1 mRNA (Figure 7A). Then, we tested whether this region of Mstn mRNA could be directly cleaved by purified recombinant IRE1 $\alpha$ protein, using a synthetic 14-nucleotide Mstn mRNA molecule labeled with 5'-FAM fluorophore and 3 '-black hole quencher (BHQ) as the substrate in an in vitro biochemical assay (Figure 7B). Incubation with IRE1 $\alpha$ of this Mstn mRNA substrate abolished the quenching effect of $\mathrm{BHQ}$, resulting in a dramatic increase in its fluorescent output signal (Figure 7B). By contrast, addition of $4 \mu 8 \mathrm{C}$, a pharmacological inhibitor of IRE1 $\alpha$ RNase activity $(51,52)$, markedly reduced this cleavage-based fluorescent signal (Figure 7B). To affirm IRE1 $\alpha$ 's action at the putative cleavage site, we generated a synthetic mutant Mstn mRNA substrate by replacing the conserved GC with $\mathrm{CA}$, which completely abolished the cleavage-based fluorescent signal in comparison with its WT version (Figure 7B). Furthermore, quantitative RT-PCR analysis in HEK293T cells showed that cotransfected expression of IRE1 $\alpha$ resulted in significantly decreased abundance of the WT but not the GC-to-CA mutant Mstn mRNA spanning the entire coding region of myostatin (Figure $7 \mathrm{C}$ ). Interestingly, RT-PCR analyses showed that this Mstn mRNA could be immunoprecipitated along with the WT but not the truncation mutant IRE1 $\alpha$ protein lacking its RNase domain from cotransfected HEK293T cells (Figure 7D); by contrast, deletion of the stem-loop structure of the Mstn mRNA abolished its ability to associate with IRE1 $\alpha$ protein (Figure 7D). This indicates the structural involvement of IRE1 $\alpha$ RNase domain and the stem-loop of the Mstn mRNA substrate in this particular RIDD machinery. We further determined whether IRE1 $\alpha$ could affect the stability of Mstn mRNA using C2C12 myotubes following treatment with actinomycin D. Knockdown of IRE1 $\alpha$ resulted in a decrease in the degradation rate of Mstn mRNA (Figure 7E), whereas IRE1 $\alpha$ overexpression significantly accelerated it (Figure 7F). Collectively, these results demonstrate that Mstn mRNA could indeed serve as a bona fide RIDD substrate of IRE1 $\alpha$. 
A

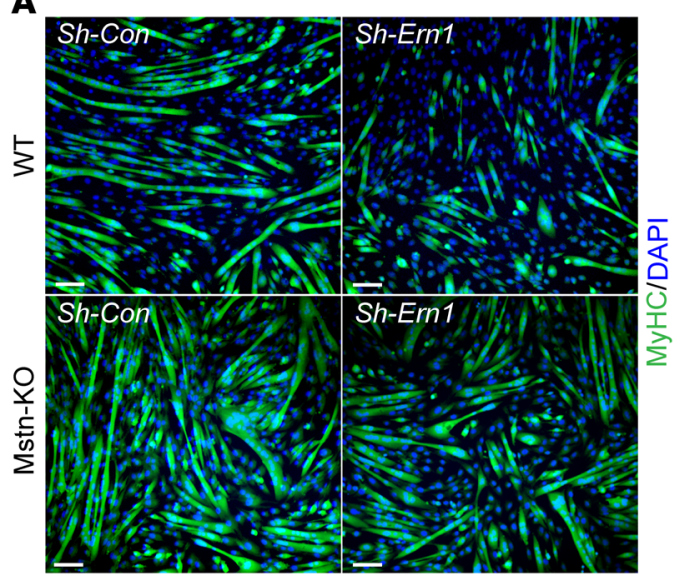

B
$\square W T+S h-C o n$
$W T+S h-E r n 1$
Mstn-KO + Sh-Con
Mstn-KO + Sh-Ern1

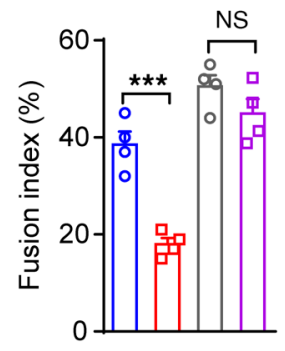

C

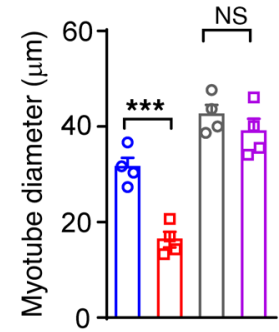

D

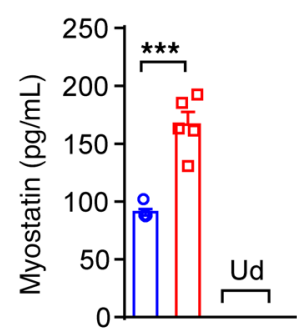

E

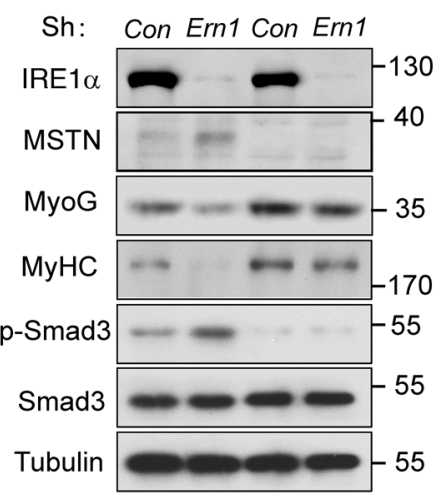

$\mathbf{F}$
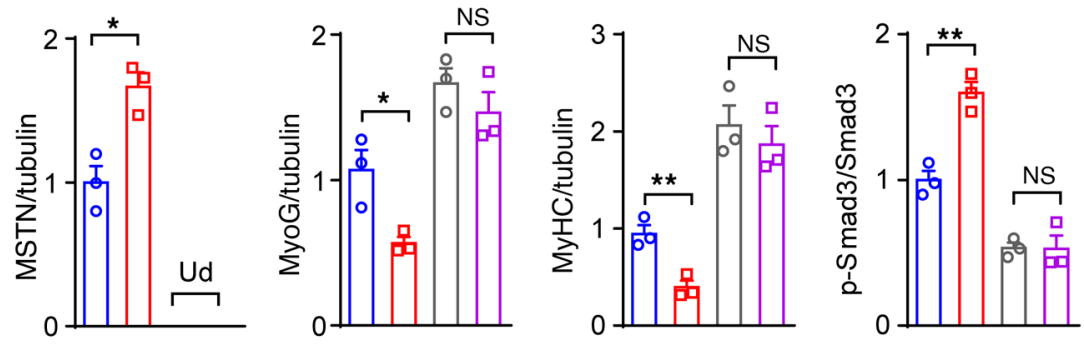

G

$\square W T+$ Sh-Con $\square$ Mstn-KO + Sh-Con
$\square W T+$ Sh-Ern1 $\square$ Mstn-KO + Sh-Ern1
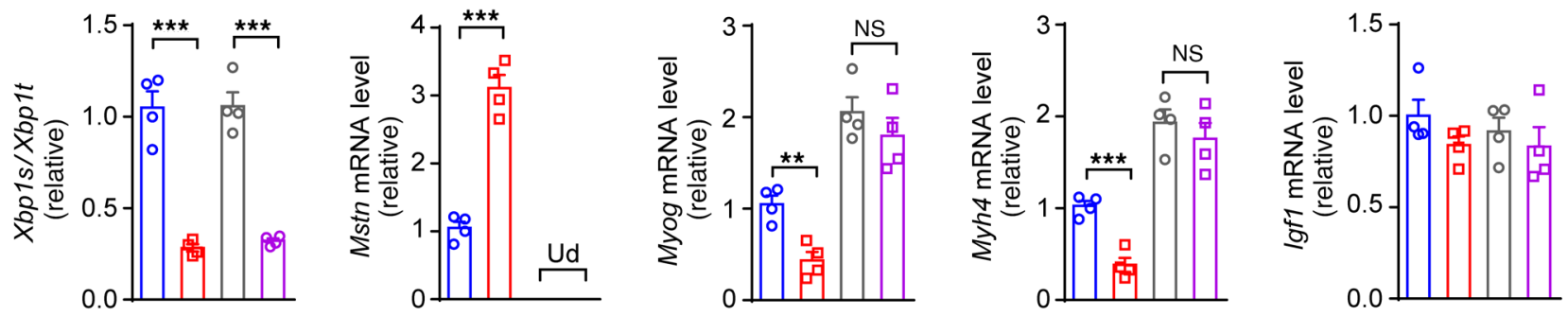

Figure 5. Myostatin ablation reverses the effect of IRE1 $\alpha$ deficiency on muscle cell differentiation and growth. WT and Mstn-KO C2C12 myoblast cells were infected for 24 hours with Sh-Con or Sh-Ern1 adenoviruses and then differentiated for 3-4 days in myogenic medium. (A-C) Representative images of MyHC immunostaining (A), and quantification of the fusion index (B) and myotube diameters (C) $(n=4$ independent experiments). (D) ELISA analysis of myostatin protein in culture medium ( $n=3$ independent experiments). ( $\mathbf{E}$ and $\mathbf{F}$ ) Immunoblot analysis of the indicated proteins (E), and quantification of averaged MSTN/tubulin, MyoG/tubulin, MyHC/tubulin, and p-Smad3/Smad3 levels (F) ( $n=3$ independent experiments). (G) Quantitative RT-PCR analysis of Xbp1 mRNA splicing and the mRNA abundance of the indicated genes $\left(n=4\right.$ independent experiments). All results represent mean $\pm S E M$. ${ }^{*} P<0.05$, ${ }^{* *} P<0.01,{ }^{* *} P<0.001$ by 2-way ANOVA with Bonferroni's multiple-comparison test. Ud, undetectable. Scale bars: $100 \mu \mathrm{m}$.

We further validated that pharmacological inhibition by $4 \mu 8 \mathrm{C}$ of IRE1 $\alpha$ RNase activity in primary myoblasts was able to, in a dose-dependent fashion, suppress Xbp1 mRNA splicing and elevate the mRNA abundance of Blos1 and Mstn (Figure 8A), leading to increased activation of myostatin signaling, reduced protein expression of myogenic markers, and prominently lower myotube formation (Figure 8, B-E). Consistently, $4 \mu 8 \mathrm{C}$ inhibition of IRE1 $\alpha$ RNase activity in differentiated C2C12 myotubes also resulted in decreased Xbp1 mRNA splicing and elevated mRNA abundance of Mstn (Figure 8F), enhanced myostatin signaling and lower levels of myogenic markers (Figure 8G), and decreased diameters of myotubes (Figure 8, $\mathrm{H}$ and I). Together, these data clearly demonstrate that IRE1 $\alpha$ could employ its RNase-dependent RIDD activity to blunt the mRNA expression of myostatin and exert its myogenic effect during the differentiation of myoblasts and hypertrophic growth of myotubes. 

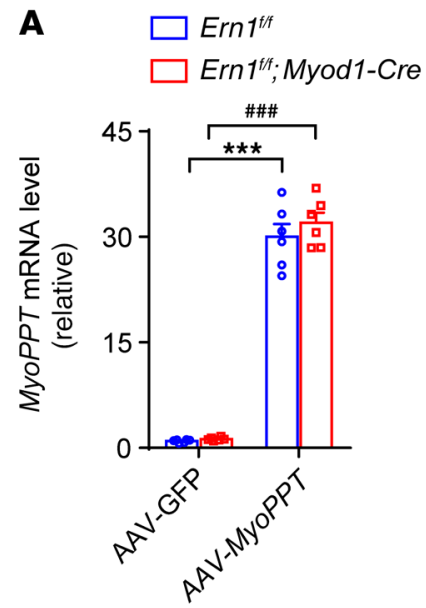

E

\section{$\square E r n 1^{f f f}$ \\ $\square$ Ern1 $1^{\text {fff }}$ Myod1-Cre}

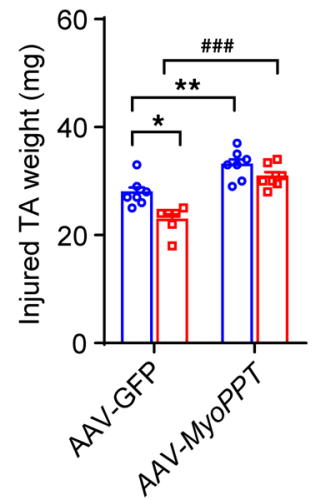

B
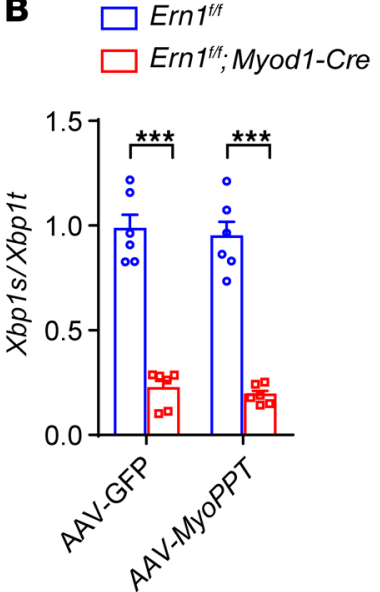

$\mathbf{F}$

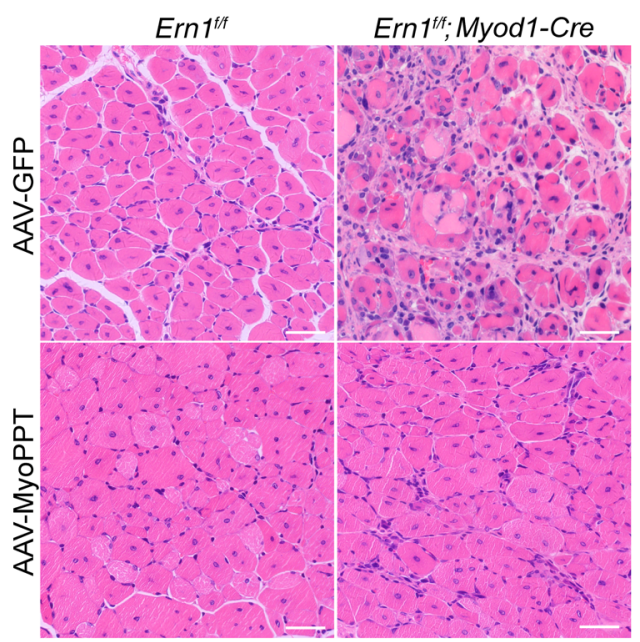

C

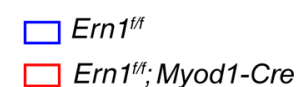

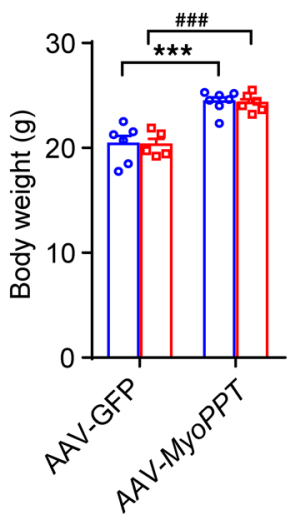

D $\square$ Ern $^{\text {th }}$

$\square$ Ern1/fifMyod1-Cre

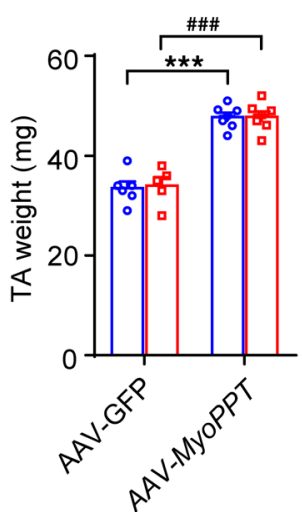

G

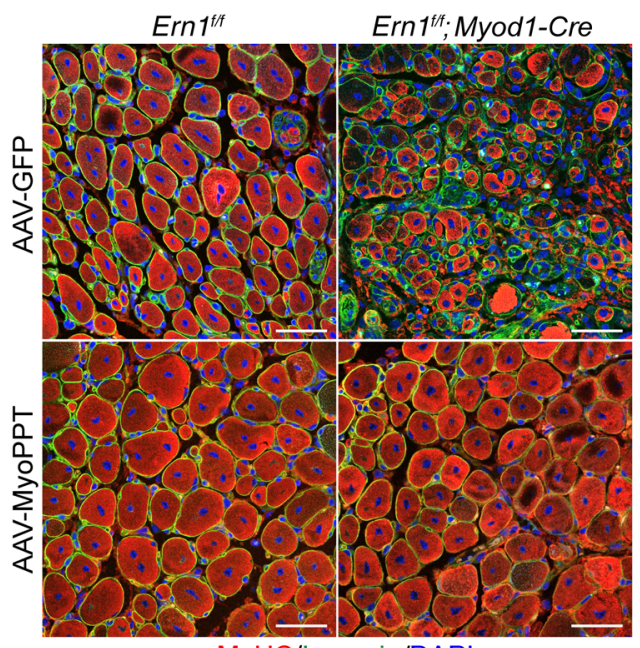

eMyHC/Lammin/DAPI

\section{I}

H

$\square E r n 1^{1 / f}+A A V-G F P$

Ern1 ${ }^{\text {fff }} \cdot$ Myod1-Cre + AAV-GFP

$\square E r n 1^{1 / f}+A A V-M y o P P T$

$\square$ Ern1 ${ }^{1 / f} ;$ Myod1-Cre + AAV-MyoPPT

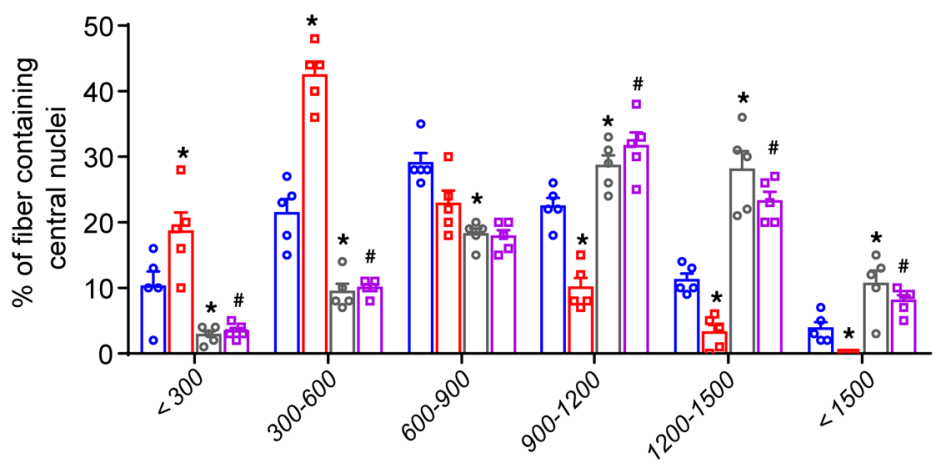

Fiber cross section area $\left(\mu \mathrm{m}^{2}\right)$

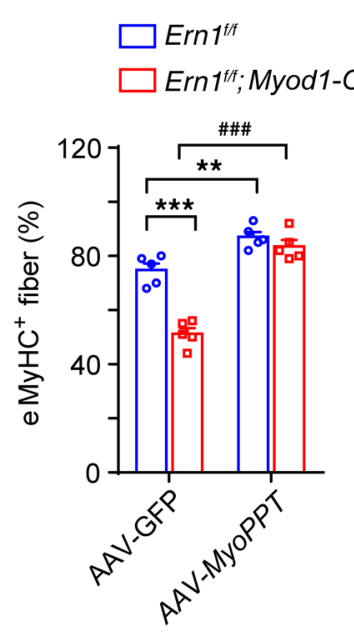


Figure 6. Inhibition in vivo of myostatin signaling rescues the impairment of muscle regeneration resulting from IRE1 $\alpha$ deficiency. Male $E r n 7^{f / f l}$ or Ern $7^{f l f l}$ Myod1-Cre mice were injected intraperitoneally on postnatal day 3 (P3) and P6 with adeno-associated virus 2/9 (AAV2/9) expressing GFP (AAV-GFP) or MyoPPT (AAV-MyoPPT). TA muscles of indicated mice were injected with PBS or CTX at 7 weeks of age and analyzed at 8 days after injection. (A and B) Quantitative RT-PCR analysis of the mRNA abundance of MyoPPT (A) and Xbp1 mRNA splicing (B) in PBS-treated uninjured TA muscles ( $n=6$ mice per group). (C) Body weight of mice of the indicated groups. (D) Weight of PBS-treated uninjured TA muscles. (E) Weight of CTX-injured TA muscles from mice of the indicated groups ( $n=5-7$ mice per group). ( $F$ and $\mathbf{G})$ Representative H\&E staining (F) and laminin (green) and eMyHC (red) immunostaining (C) of injured TA muscles ( $n=5$ mice per group). (H) Percentage of regenerated myofibers in the indicated cross-sectional areas of TA muscles. Myofibers containing centralized nuclei were quantified by Image from 500 myofibers in each mouse ( $n=5$ mice per group). (I) Quantification of the percentage of eMyHC+ myofibers within laminin staining ( $n=5$ mice per group). All data are presented as mean \pm SEM. Significance was calculated by 2-way ANOVA with Bonferroni's multiple-comparison test. ${ }^{*} P<0.05,{ }^{* *} P<0.01,{ }^{* * *} P<0.001$ vs. Ern ${ }^{I l / f l}+$ AAV-GFP. ${ }^{\#} P<0.05$, ${ }^{\# \# P}<$ 0.001 vs. Ern $7^{f / f l}$ Myod1-Cre + AAV-GFP. Scale bars: $100 \mu \mathrm{m}$.

Loss of IRE1 $\alpha$ exacerbates the dystrophic phenotypes in mdx mice. DMD is the most common and severe form of muscular dystrophy, and it remains incurable to date $(33,34)$. The $m d x$ mouse model of DMD exhibits extensive muscle degeneration and regeneration due to a null mutation in the Dmd gene, coding for the dystrophin protein, that causes higher sensitivity of myofibers to contractile stress (53). We wondered whether the IRE1 $\alpha$-myostatin regulatory circuit also plays a protective role in the setting of muscular dystrophy. To this end, we generated $m d x / E r n 1^{f / / f l}$ Myod1-Cre mice with muscle-specific IRE1 $\alpha$ deletion in the $m d x$ background through genetic intercrossing. As expected, we detected significantly increased abundance of TA muscle Mstn mRNA as well as higher circulating myostatin protein in $m d x / E r n 1^{f / f l}$ Myod1-Cre mice relative to their $m d x / E r n 1^{f l / f l}$ littermates (Figure 9, A and B). This was associated with elevated Smad3 phosphorylation and reduced eMyHC protein level (Figure 9, C and D). Supporting a defective IRE1 $\alpha$-mediated suppression of myostatin and weakened muscle regeneration, $m d x / E r n 1^{f / f l} \mathrm{Myod1}$-Cre mice at 6 weeks, as well as at 21 weeks, of age had grossly smaller gastrocnemius (Gas) and TA muscles, and the weight of these muscles was significantly lower than in their $m d x / E r n 1^{f / f l}$ counterparts (Figure 9, E and F, and Supplemental Figure 9A). Histological analyses revealed more severe muscle dystrophic characteristics in $\operatorname{mdx} /$ Ern $^{f l / f l}$ Myod1Cre animals, as indicated by higher variations in their fiber size, apparently elevated number of small fibers, and more infiltration of inflammatory immune cells as a result of degenerated myofibers (Figure 9G and Supplemental Figure 9B). Immunostaining of laminin in muscle sections showed many smaller regenerating myofibers in Gas and TA muscles of $m d x /$ Ern $^{\text {fl/fl }}$ Myod1-Cre mice at 6 or 21 weeks of age compared with their $m d x / E r n 1^{f / f l}$ counterparts (Figure $9 \mathrm{H}$ and Supplemental Figure 9C), with a significantly lower percentage of larger regenerating myofibers observed in TA muscles (Figure 9I and Supplemental Figure 9D). This indicates that loss of IRE1 $\alpha$ results in impaired maturation of myofibers during the regenerative growth of $m d x / E r n 1^{f l / f l}$ Myod1Cre muscles, similar to its effect on reparative muscle growth following CTX-induced acute injury. Using Evans blue dye staining to assess muscle fiber integrity, we further detected more pronounced damage with higher dye uptake in $m d x / E r n 1^{f l / f l}$ Myod1Cre muscles (Figure 9, J and K). In addition, we observed no overt changes in the serum levels of creatine kinase (CK), a hallmark indicator of damaged muscles, in Ern $1^{\text {flfl }}$ Myod1-Cre mice, but found marked elevations in serum CK levels in $m d x / E r n 1^{f / f l}$ mice relative to their $E r n 1^{f / f l}$ controls (Figure 9L). Moreover, $m d x / E r n 1^{f / f f l}$ Myod1-Cre mice displayed more pronounced increases in their serum CK levels relative to $m d x / E r n 1^{f / f l}$ mice $(24,109 \pm 3797 \mathrm{U} / \mathrm{L}$ vs. $14,437 \pm 2719 \mathrm{U} / \mathrm{L}, n=5$ mice per genotype, $P=0.0013)$. To further evaluate the impact of IRE1 $\alpha$ deficiency on muscle functionality and performance, we challenged the animals with treadmill exercise and measured the running time and distance before exhaustion. Whereas Ern ${ }^{f l / f l}$ Myod1-Cre and Ern ${ }^{f l / f l}$ mice displayed no significant difference in their exercise performance, $m d x /$ $E r n 1^{f / f l}$ animals showed a significantly shorter running time and distance (Figure 9M), and $m d x / E r n 1^{f / f l}$ Myod1-Cre mice exhibited a more pronounced impairment in their running ability $(m d x /$ Ern1 $^{f / f l}, 651 \pm 88 \mathrm{~m}$, vs. $m d x /$ Ern1 $^{f / / f l}$ Myod1-Cre, $364 \pm 90 \mathrm{~m} ; n=$ 5 mice per genotype, $P=0.004)$. These results demonstrate that ablation of IRE1 $\alpha$ aggravates the severity of muscle dystrophy stemming from the lack of dystrophin in $m d x$ mice, further establishing the IRE1 $\alpha$-myostatin axis as an important regulatory loop during regeneration in the setting of muscle dystrophy.

\section{Discussion}

Skeletal muscle relies critically on its self-regeneration for preserving muscle mass and ensuring muscle function in response to injury and disease. Muscle regenerative capacity declines with age and is severely compromised in many muscular dystrophies (39). Delineation of the molecular mechanisms involved in regulating skeletal muscle regeneration and repair is of great importance to development of therapeutic approaches for muscle degenerative diseases. Herein, using 2 mouse models, we have uncovered a critical role for the UPR sensor IRE1 $\alpha$ in promoting skeletal muscle regeneration in response to CTX-induced acute muscle injury or genetic muscle dystrophy. Ablation of IRE1 $\alpha$ specifically in skeletal muscles results in impaired muscle regenerative repair or worsened progression of muscle dystrophy. Importantly, our data demonstrate that IRE1 $\alpha$ employs its RNase-dependent RIDD action to downregulate the level of mRNA encoding myostatin, a key inhibitory regulator of muscle growth and repair. Our findings reveal a physiological function of the RIDD output of IRE1 $\alpha$ enzyme in the control of muscle regeneration and growth (Figure 10), suggesting that IRE1 $\alpha$ may serve as an important modifier in the disease progression of muscular dystrophy or muscle loss.

IRE1 $\alpha$ is the most ancient ER stress sensor, conveying a critical signaling response through its RNase activity to generate an active transcription factor, XBP1s $(3,5)$. While IRE1 $\alpha$ is best known to exert its prosurvival actions through XBP1s when coping with ER stress, emerging lines of evidence also indicate that activated IRE1 $\alpha$ RNase activity may have a broad range of functions, as reflected by an increasing number of identified putative RIDD substrates $(8,15)$. However, the physiologically relevant RIDD actions of IRE1 $\alpha$ have yet to be established in vivo. In this study, we provide physiological evidence for a RIDD-mediated mechanism by which IRE1 $\alpha$ regulates myostatin production and 
A

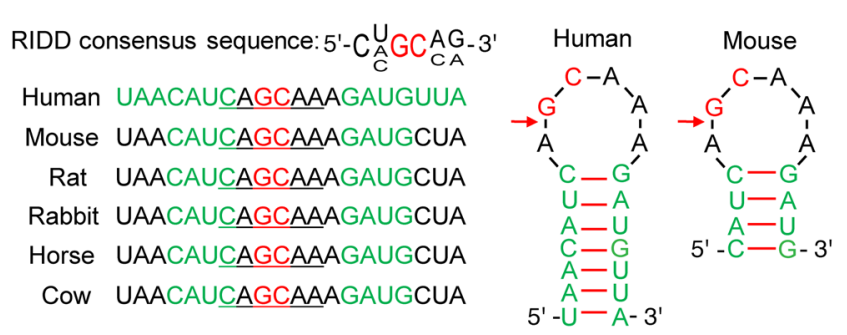

B

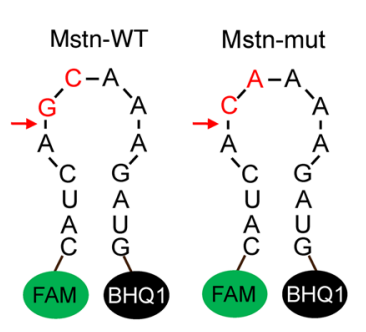

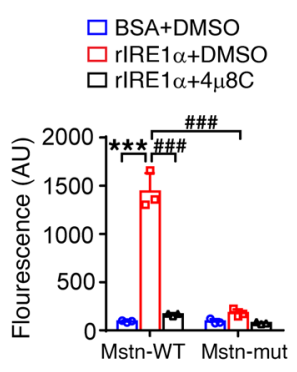

C

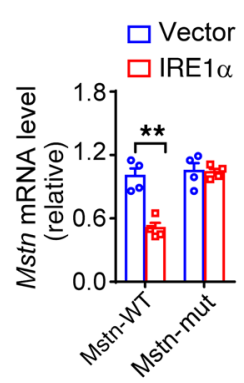

D

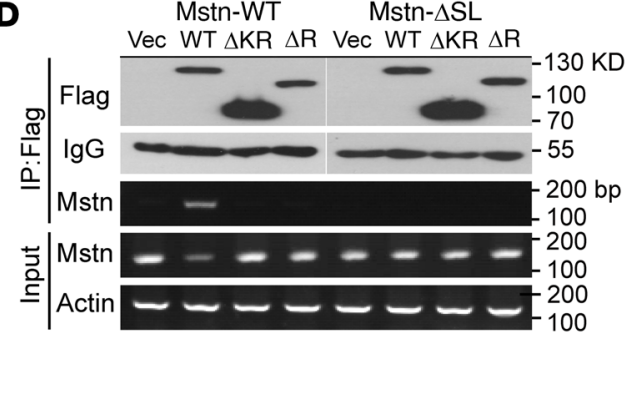

E

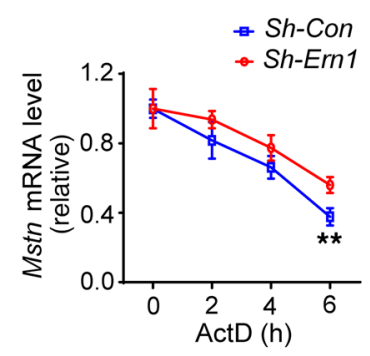

$\mathbf{F}$

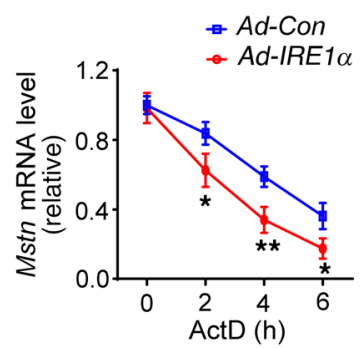

Figure 7. Myostatin mRNA is a RIDD target of IRE1 $\alpha$. (A) Sequence alignment of Mstn mRNAs from the indicated species with the putative conserved RIDD region, with the predicted stem-loop secondary structure shown for human and mouse Mstn mRNA. The potential IRE1 $\alpha$ cleavage site is indicated by an arrow. (B) Fluorescence-based analysis of IRE1 $\alpha$ cleavage of the synthetic WT or mutant (mut, GC to CA) Mstn mRNA ( $n=3$ independent experiments). (C) Quantitative RT-PCR analysis of the abundance of Mstn mRNA in HEK293T cells cotransfected with plasmids expressing the entire coding region of WT or mutant myostatin (GC to CA) together with empty vector or human IRE1 $\alpha$ expression plasmid ( $n=4$ independent experiments). (D) HEK293T cells were cotransfected with plasmids expressing the WT or stem-loop deletion ( $\triangle \mathrm{SL}$ ) mutant myostatin together with vector (Vec) control or plasmids expressing FLAG-tagged human WT or deletion mutant (KR, kinase and RNase domain; R, RNase domain) IRE1 $\alpha$ proteins. Representative RT-PCR analysis of Mstn mRNA from immunoprecipitates (IP) using anti-FLAG antibody or from total cellular RNA (Input). Shown also are representative immunoblots of immunoprecipitated IRE1 $\alpha$ proteins and IgG control ( $n=3$ independent experiments). (E and F) The stability of the Mstn mRNA was determined by quantitative RT-PCR in C2C12 myotubes infected with Sh-Con or Sh-Ern1 adenoviruses (E), or Ad-Con or Ad-IRE1 $\alpha$ adenoviruses (F), after treatment with actinomycin D (ActD) for the indicated time intervals. Data are shown relative to the value at time 0 of ActD treatment (set as 1 ) after normalization to the GAPDH mRNA level as internal control ( $n=3$ independent experiments). All data are shown as mean \pm SEM. Significance was calculated by 2 -way ANOVA with Bonferroni's multiple-comparison test. ${ }^{*} P<0.05,{ }^{* *} P<0.01,{ }^{* *} P<0.001$. ${ }^{\# \#} P<0.001$. Scale bars: $100 \mu \mathrm{m}$.

regenerative maturation of myofibers in response to muscle injury or degenerative dystrophy. It is notable that ablation of IRE1 $\alpha$ did not cause changes in muscle and myofiber sizes or myostatin expression levels in Ern1 ${ }^{t / f l}$ Myod1-Cre mice in the absence of injury or dystrophy. Moreover, IRE1 $\alpha$ phosphorylation is relatively low in muscle cells under basal conditions, but is highly induced during myoblast differentiation or upon muscle injury. This suggests that activation of the IRE1 $\alpha$-myostatin regulatory circuit is coupled to the stress condition induced by muscle injury or damage, conceivably reflecting the multifunctional property of IRE1 $\alpha$ in utilizing its RIDD activity to handle various types of stress conditions in a tissue- or cell type-specific fashion.

It is worth noting that we used Myod1-Cre mice to create mice with IRE1 $\alpha$ deletion specifically in skeletal muscles. Because of the activated expression pattern of Myod1 during myogenesis (54), IRE1 $\alpha$ abrogation most likely occurred in muscle stem cells (i.e., satellite cells) at particular differentiation stages as well as in mature muscle fibers. Our results from analyses of muscle cell differentiation and growth in vitro suggest that IRE1 $\alpha$ can promote both myoblast differentiation and myotube hypertrophy growth. Thus, the impairment of injury-induced regeneration in Ern $1^{f / f l}$ Myod1-Cre mice can be largely attributed to the cell-autonomous action of IRE1 $\alpha$ in myocyte differentiation and growth. This is in accordance with the fact that myostatin can regulate both differentiation in myoblasts and hypertrophy in myotubes (55). Moreover, inhibition of myostatin has been shown to improve the hallmarks of the muscular dystrophy in $m d x$ mice $(56,57)$. This also supports our model that the lack of IRE1 $\alpha$-myostatin regulatory axis contributes to the more severe dystrophic phenotypes in $m d x /$ Ern1 $^{f l / f l}$ Myod1-Cre mice.

$\mathrm{XBP} 1$ has been recently reported to be dispensable for satellite cell-mediated muscle regeneration, whereas the PERK/ eIF $2 \alpha$ arm of the UPR may play an important role in this process (58). Documented studies have also suggested that myostatin can suppress satellite cell activation and self-renewal (43, 59, 60). While our results indicate that IRE1 $\alpha$ deficiency caused no significant changes in the number of TA muscle satellite cells upon acute injury, it has yet to be more thoroughly interrogated whether IRE1 $\alpha$ can regulate satellite cells through a myostatin-mediated mechanism or cell-intrinsically in an XBP1independent manner, e.g., by affecting the STAT3 pathway (35, 61 , which was reported to critically regulate the proliferation and self-renewal of muscle stem cells (62).

Myostatin, GDF11, and activin A are the 3 circulating factors that belong to the TGF- $\beta$ family in the negative control of skeletal muscle mass (30). It remains to be dissected whether IRE1 $\alpha$ also 
$\begin{array}{ll}\text { A } & \square \text { DMSO } \\ & \square 4 \mu \mathrm{CC}-5 \mu \mathrm{M} \\ & \square 4 \mu \mathrm{CC}-10 \mu \mathrm{M}\end{array}$
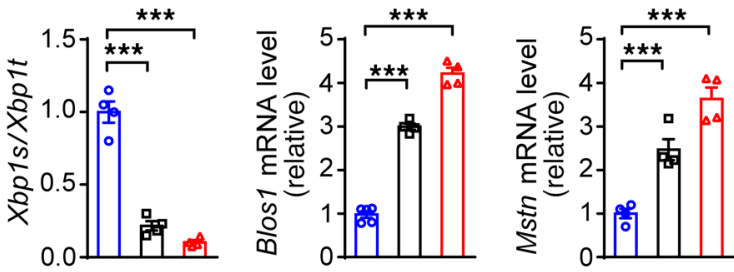

B

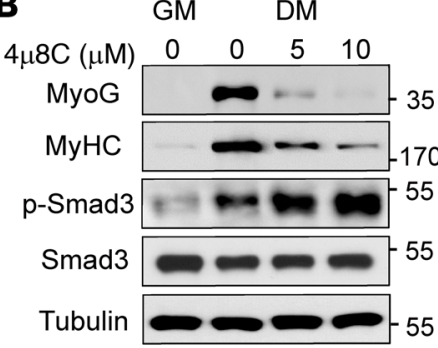

E
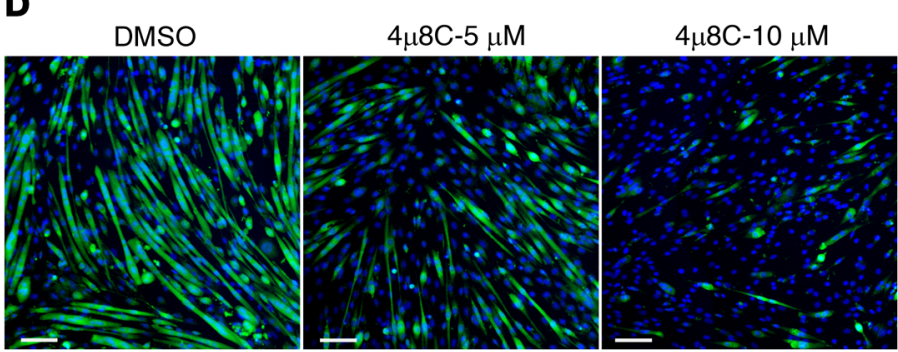

MyHC/DAPI

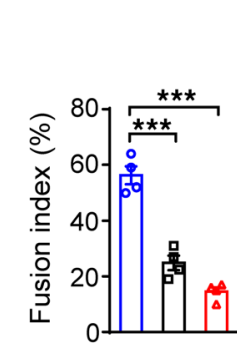

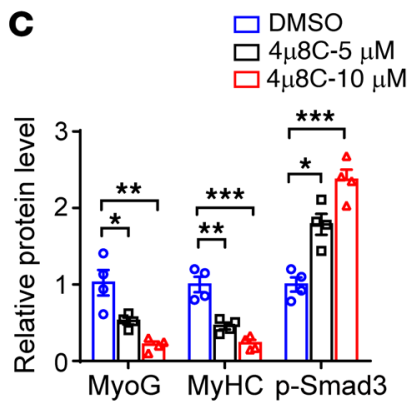

G

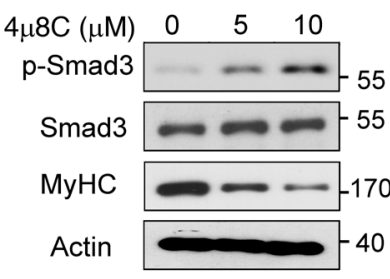

H

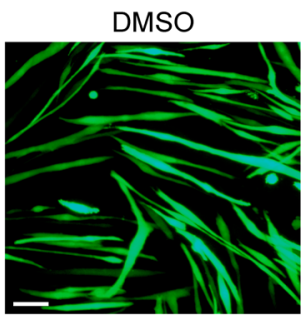

$4 \mu 8 \mathrm{C}-5 \mu \mathrm{M}$

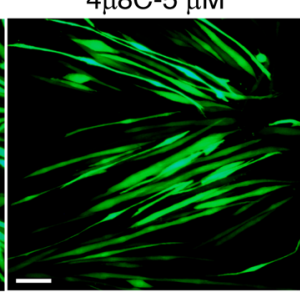

$4 \mu 8 \mathrm{C}-10 \mu \mathrm{M}$

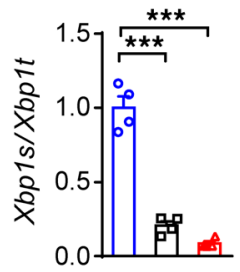

$\square$ DMSO

$\square 4 \mu 8 \mathrm{C}-5 \mu \mathrm{M}$ $\square 4 \mu 8 \mathrm{C}-10 \mu \mathrm{M}$

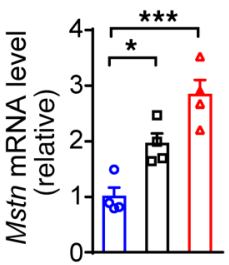

Figure 8. Pharmacological inhibition of IRE1 $\alpha$ RNase activity impairs muscle cell differentiation and growth. (A-E) Primary myoblasts isolated from WT mice were cultured in growth medium (GM), and subsequently incubated in differentiation medium (DM) with or without $4 \mu 8 \mathrm{C}$ for 3 days ( $n=4$ independent experiments). (A) Quantitative RT-PCR analysis of Xbp1 mRNA splicing and the mRNA abundance of Blos1 and Mstn. (B) Immunoblot analysis of the expression of the indicated proteins. (C) Quantification of MyoG/tubulin, MyHC/tubulin, and p-Smad3/Smad3 levels. (D) MyHC immunostaining. (E) Quantification of the fusion index. (F and $\mathbf{G}$ ) Differentiated C2C12 myotubes were treated for 24 hours with DMSO versus $5 \mu \mathrm{M}$ or $10 \mu \mathrm{M} 4 \mu 8 \mathrm{C}$. (F) Quantitative RT-PCR analysis of Xbp1 mRNA splicing and the Mstn mRNA abundance ( $n=4$ independent experiments). (C) Immunoblot analysis of the indicated proteins ( $n=3$ independent experiments). ( $\mathbf{H}$ and $\mathbf{~ I ) ~ C 2 C 1 2 ~ m y o b l a s t s ~ i n f e c t e d ~ b y ~ E G F P - e x p r e s s i n g ~ a d e n o v i r u s ~ w e r e ~ d i f f e r e n t i a t e d ~ f o r ~} 4$ days and likewise treated with $4 \mu 8 \mathrm{C}$ ( $n=4$ independent experiments). (H) Representative images of myotubes by fluorescence microscopy. (I) Quantification of myotube diameters using Image software. All data are shown as mean \pm SEM. Significance was calculated by 1-way ANOVA with Bonferroni's multiple-comparison test. ${ }^{*} P<0.05,{ }^{* *} P<0.01,{ }^{* *} P<0.001$. Scale bars: $100 \mu \mathrm{m}$.

contributes in the regulation of GDF11 or activin A production. In addition, even though IRE1 $\alpha$ did not directly, or through myostatin signaling, affect the expression of IGF1, a potent inducer of muscle hypertrophy (30), in differentiated myotubes, and XBP1s showed a merely marginal effect on myotube growth in vitro, it warrants further investigation whether IRE1 $\alpha$ can simultaneously act to positively regulate Igfl expression through the XBP1s pathway in vivo.

In summary, our results have unmasked a crucial role of IRE1 $\alpha$ in regulating skeletal muscle regeneration through RIDD control of myostatin expression. Given its well-established central role in suppressing muscle regeneration and growth $(30,42,43,59,60$, 63-65), myostatin has been shown to represent a valuable target for improving muscular dystrophy in mouse models. In this scenario, targeted modulation of the RIDD output of IRE1 $\alpha$ may represent a potential avenue for therapeutic intervention in DMD or other skeletal muscle degenerative diseases.

\section{Methods}

Detailed methods can be found in Supplemental Methods.

Animal studies. C57BL/6J mice were obtained from Shanghai Laboratory Animal Co. Ltd. Generation of Ern $1^{f l / f l}$ mice has been described previously in detail (38). Ern $1^{f / f l}$ mice were intercrossed with mice expressing Cre recombinase under the control of a Myod1 promoter (The Jackson Laboratory, stock 014140) to create mice with muscle-specific deletion of IRE1 $\alpha$. The $m d x$ mice were purchased from The Jackson Laboratory (stock 000476). Male Ern1 $1^{f / f l}$ mice were first bred with homozygote female $D m d^{m d x / m d x}$ mice to generate $E r n 1^{f /+} D m d^{m d x / Y}$ males and $E r n 1^{f /+} D m d^{m d x /+}$ females. Male $E r n 1^{f l /+} D m d^{m d x / Y}$ mice were then bred to female $D m d^{m d x / m d x}$ mice to generate $E r n 1^{f l /+} D m d^{m d x / m d x}$ females and $E r n 1^{f /+} D m d^{m d x / Y}$ males, which were subsequently intercrossed to obtain Ern1 $1^{f / f l} D m d^{m d x / m d x}$ females. Female $E r n 1^{f / f l} D m d^{m d x / m d x}$ mice were finally bred with

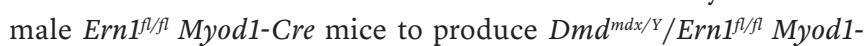


A

$\square m d x / E r n 1^{t / 1}$

$\square \mathrm{mdx} /$ Ern ${ }^{1 f f} ;$ Myod1-Cre

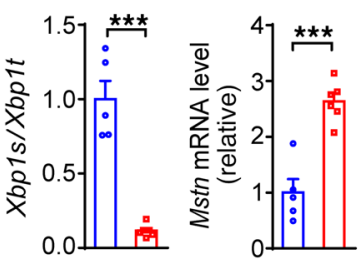

E

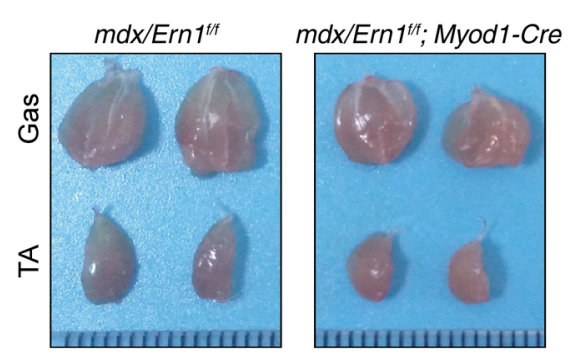

B

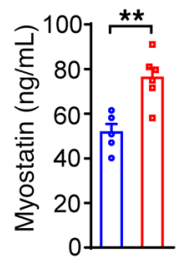

$\square m d x / E r n 1^{t f f}$

$\square \mathrm{mdx} /$ Ern 1 ${ }^{\text {thr }}$ Myod1-Cre
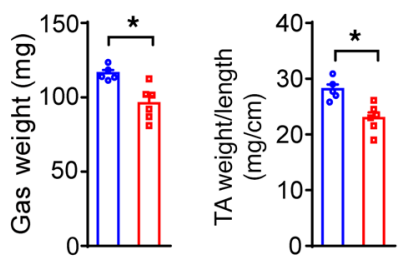

D

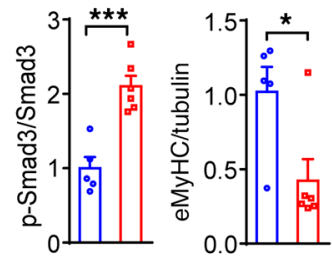

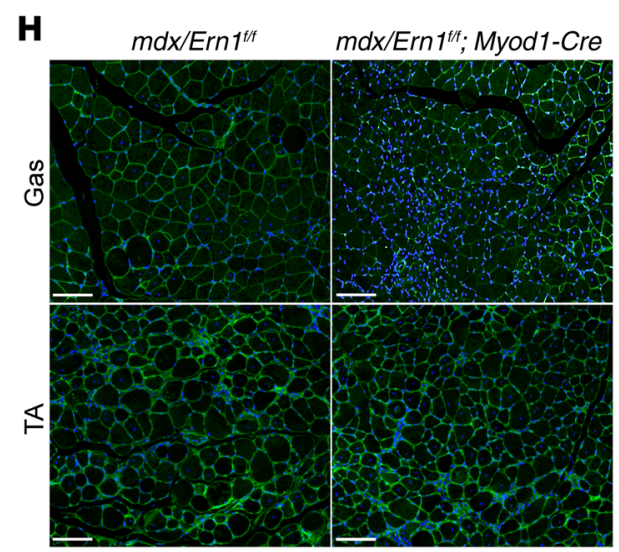

Laminin/DAPI

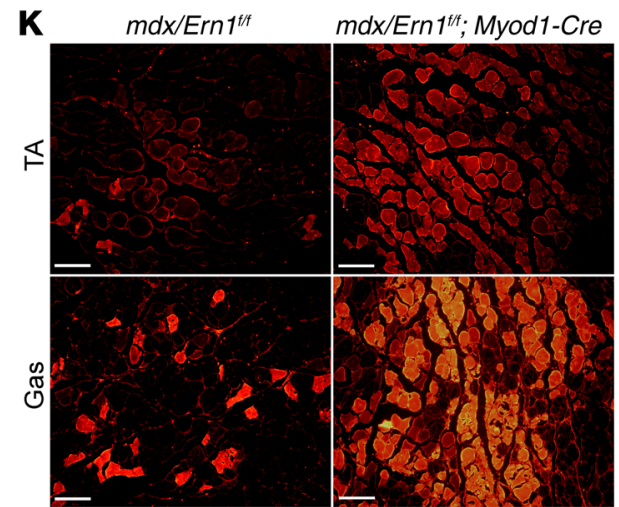

I

$\square m d x / E r n 1^{t t}$

$\square m d x / E r n 1^{t r} ;$ Myod1-Cre

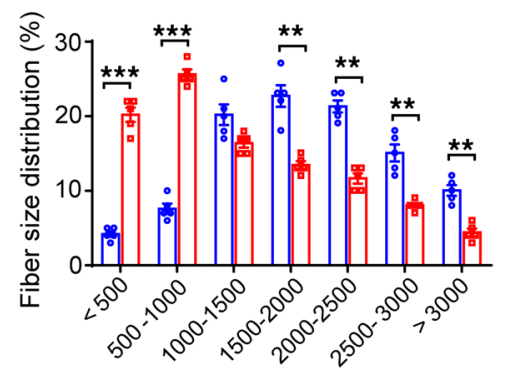

Fiber cross section area $\left(\mu \mathrm{m}^{2}\right)$
G $\quad m d x / E r n 1^{t h}$ mdx/Ern1 ${ }^{\text {tit }}$; Myod1-Cre

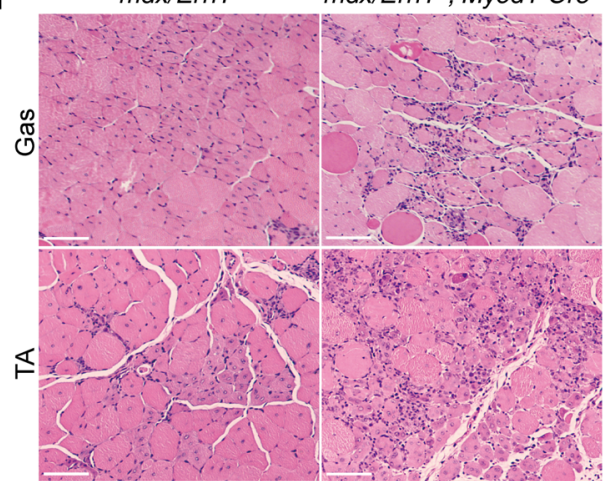

J

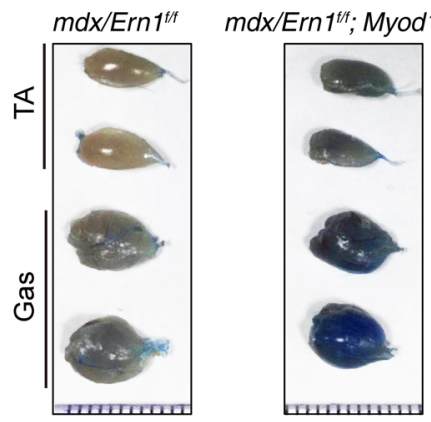

$\mathbf{L}$

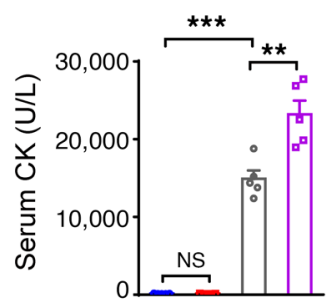

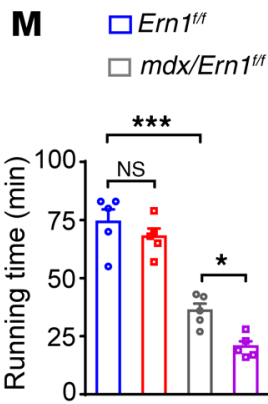

$\square$ Ern 1"; Myod1-Cre

$\square$ mdx/Ern 1"t; Myod1-Cre

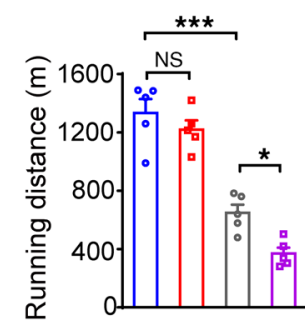


Figure 9. Loss of IRE1 $\alpha$ exacerbates the dystrophic phenotypes in $\mathbf{m d x}$ mice. (A) Quantitative RT-PCR analysis of Xbp1 mRNA splicing and the mRNA abundance of Mstn in TA muscles from male $m d x / E r n 7^{f / f l}$ and $m d x / E r n f^{f l / f l}$ Myod1-Cre mice at 6 weeks of age $(n=5-6$ mice per genotype). (B) ELISA analysis of serum myostatin levels ( $n=5-6$ mice per genotype). (C) Immunoblot analysis of TA muscle lysates using the indicated antibodies. (D) Averaged $\mathrm{p}-\mathrm{Smad} 3 / \mathrm{Smad} 3$ and eMyHC/tubulin ratios after normalization to the value in $m d x$ muscle $(n=5-6$ mice per genotype). (E and $\mathbf{F}$ ) Representative pictures (E) and weight (F) of gastrocnemius (Gas) and TA muscles from $m d x / E r n 1^{f / f l}$ and $m d x / E r n 1^{f / f l}$ Myod1-Cre mice at 6 weeks of age ( $n=5-6$ mice per genotype). (G and $\mathbf{H}$ ) Representative images of H\&E staining of Gas and TA muscles (G) and laminin (green) immunostaining of Gas and TA muscles (H) ( $n=5$ mice per genotype). (I) Percentage of myofibers in the indicated cross-sectional areas of TA muscle. Quantification was conducted by Image from 500 myofibers of the TA muscle from each mouse ( $n=5$ mice per genotype). (J and $\mathbf{K}$ ) Analysis of Evans blue dye uptake of Gas and TA muscles ( $n=$ 5 mice per genotype). (J) Representative images of Gas and TA muscles. (K) Representative fluorescent micrographs of muscle sections. ( $L$ and M) Serum creatine kinase (CK) activity (L) and mean running time and distance on a motorized treadmill (M) for mice of the indicated genotypes at 6 weeks of age ( $n=5$ mice per genotype). All data are shown as mean \pm SEM. Significance was calculated by unpaired 2-tailed Student's $t$ test (A, B, D, and $\mathbf{F}$ ), 2-way ANOVA (I), or 1-way ANOVA ( $\mathbf{L}$ and $\mathbf{M}$ ) with Bonferroni's multiple-comparison test. ${ }^{*} P<0.05,{ }^{* *} P<0.01,{ }^{* *} P<$ 0.001 . Scale bars: $100 \mu \mathrm{m}$.

Cre or $D m d^{m d x / Y} / E r n 1^{f l / f l}$ males. Male offspring were genotyped, randomly assigned, and used at 5-21 weeks of age. Littermate controls were used in all cases, and histological analysis was performed by investigators who were blinded to the experimental groups.

Statistics. All data represent the mean SEM, with $P$ less than 0.05 considered as a statistically significant difference. Differences between groups were tested using the unpaired 2-tailed Student's $t$ test, or by ANOVA with Bonferroni's post hoc test when more than 2 groups were compared, as indicated in the figure legends. All data points were used in statistical analyses. Statistical analyses were carried out using Prism 8 software (GraphPad Software).
Study approval. All animal studies were carried out in strict accordance with the institutional guidelines for the humane treatment of animals, and all experimental protocols were approved by the IACUCs at the College of Life Sciences, Wuhan University, and the Model Animal Research Center of Nanjing University.

\section{Author contributions}

$\mathrm{SH}, \mathrm{ZG}$, and YL conceived and designed the studies. SH performed most of the experiments and analyzed the data. TF, YY, QL, LL, Jing Liu, XZ, QZ, QG, DX, and Yong Chen conducted some of the animal studies and cellular experiments. SH, ZG, and YL analyzed data. XW, Yulin Chen, and Jianmiao Liu provided assistance with the experimental design, reagents and data analysis. SH, ZG, and YL wrote the manuscript.

\section{Acknowledgments}

We thank Dahai Zhu, Chinese Academy of Medical Sciences, and Zhen-Guo Wu, Hong Kong University of Science and Technology, for insightful discussions. This work was supported by grants from the Ministry of Science and Technology of China (National Key R\&D Program of China 2018YFA0800700 and 2016YFA0500100) to YL and ZG, and from the National Natural Science Foundation of China (31690102, 91857204, 32021003, 31922033, 91857105, 31771291, 91739303, and 32071136) to YL, ZG, Jianmiao Liu, and TF; and by the Natural Science Foundation of Jiangsu Province (BK20170014, to ZG) and Fundamental Research Funds for the Central Universities (021414380511 and 2042020kf1056, to ZG and YL).

Address correspondence to: Yong Liu, College of Life Sciences, Wuhan University, 299 Bayi Road, Wuhan 430072, China. Phone: 86.27.68753463; Email: liuyong31279@whu.edu.cn. Or to: Zhenji Gan, Model Animal Research Center of Nanjing University, 12 Xuefu Road, Pukou, Nanjing 210061, China. Phone: 86.25.58641546; Email: ganzj@nju.edu.cn.

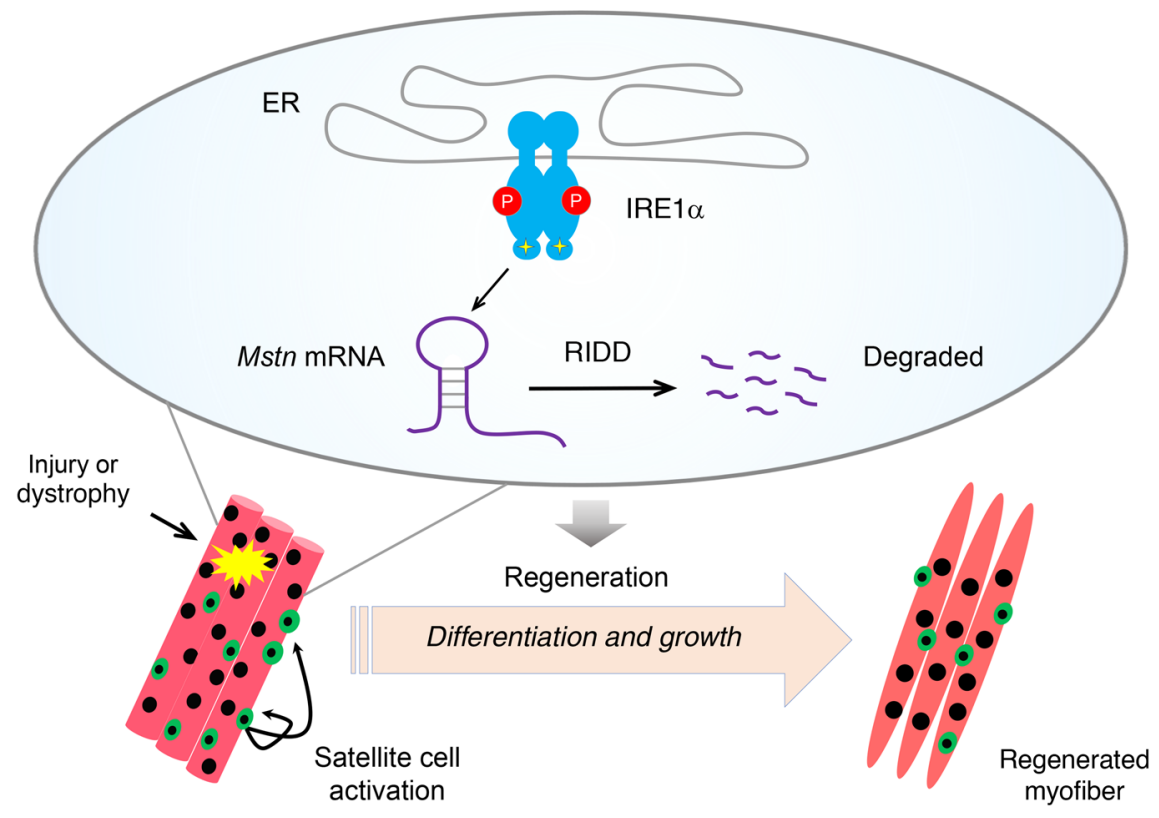

Figure 10. IRE1 $\alpha$ regulates skeletal muscle regeneration through RIDD suppression of myostatin. Upon injury-induced or dystrophy-associated stress, IRE1 $\alpha$ RNase is activated in muscle cells to decay the mRNA encoding myostatin, thereby promoting myoblast differentiation and myotube growth during the skeletal muscle regeneration response. 
1. Hetz C, Glimcher LH. Protein homeostasis networks in physiology and disease. Curr Opin Cell Biol. 2011;23(2):123-125.

2. Schroder M, Kaufman RJ. The mammalian unfolded protein response. Annu Rev Biochem. 2005;74:739-789.

3. Walter P, Ron D. The unfolded protein response: from stress pathway to homeostatic regulation. Science. 2011;334(6059):1081-1086.

4. Cox JS, et al. Transcriptional induction of genes encoding endoplasmic reticulum resident proteins requires a transmembrane protein kinase. Cell.1993;73(6):1197-1206.

5. Han J, Kaufman RJ. Physiological/pathological ramifications of transcription factors in the unfolded protein response. Genes Dev. 2017;31(14):1417-1438

6. Yoshida $\mathrm{H}$, et al. XBP1 mRNA is induced by ATF6 and spliced by IRE1 in response to ER stress to produce a highly active transcription factor. Cell. 2001;107(7):881-891.

7. Hollien J, et al. Regulated Ire1-dependent decay of messenger RNAs in mammalian cells. J Cell Biol. 2009;186(3):323-331.

8. Hollien J, Weissman JS. Decay of endoplasmic reticulum-localized mRNAs during the unfolded protein response. Science. 2006;313(5783):104-107.

9. Oikawa D, et al. Identification of a consensus element recognized and cleaved by IRE1 alpha. Nucleic Acids Res. 2010;38(18):6265-6273.

10. Hetz C, Papa FR. The unfolded protein response and cell fate control. Mol Cell. 2018;69(2):169-181.

11. Hetz C. The unfolded protein response: controlling cell fate decisions under ER stress and beyond. Nat Rev Mol Cell Biol. 2012;13(2):89-102.

12. Hotamisligil GS. Endoplasmic reticulum stress and the inflammatory basis of metabolic disease. Cell. 2010;140(6):900-917.

13. Hetz C, et al. Mechanisms, regulation and functions of the unfolded protein response. Nat Rev Mol Cell Biol. 2020;21(8):421-438.

14. Bettigole SE, Glimcher LH. Endoplasmic reticulum stress in immunity. Annu Rev Immunol. 2015;33:107-138.

15. Maurel M, et al. Getting RIDD of RNA: IRE1 in cell fate regulation. Trends Biochem Sci. 2014;39(5):245-254.

16. Lee AH, et al. Dual and opposing roles of the unfolded protein response regulated by IRE1alpha and XBP1 in proinsulin processing and insulin secretion. Proc Natl Acad Sci US A. 2011;108(21):8885-8890.

17. So JS, et al. Silencing of lipid metabolism genes through IRE1 $\alpha$-mediated mRNA decay lowers plasma lipids in mice. Cell Metab. 2012;16(4):487-499.

18. Osorio F, et al. The unfolded-protein-response sensor IRE- $1 \alpha$ regulates the function of CD $8 \alpha+$ dendritic cells. Nat Immunol. 2014;15(3):248-257.

19. Zhao N, et al. Pharmacological targeting of MYC-regulated IRE1/XBP1 pathway suppresses MYC-driven breast cancer. JClin Invest. 2018;128(4):1283-1299.

20. Shan B, et al. The metabolic ER stress sensor IRE1 $\alpha$ suppresses alternative activation of macrophages and impairs energy expenditure in obesity. Nat Immunol. 2017;18(5):519-529.
21. Sha H, et al. Stressed out about obesity: IRE1 $\alpha$ XBP1 in metabolic disorders. Trends Endocrino Metab. 2011;22(9):374-381.

22. Huang $\mathrm{S}$, et al. Emerging roles for the ER stress sensor IRE1 $\alpha$ in metabolic regulation and disease. J Biol Chem. 2019;294(49):18726-18741.

23. Le Grand F, Rudnicki M. Satellite and stem cells in muscle growth and repair. Development. 2007;134(22):3953-3957.

24. Tedesco FS, et al. Repairing skeletal muscle: regenerative potential of skeletal muscle stem cells. J Clin Invest. 2010;120(1):11-19.

25. Ten Broek RW, et al. Regulatory factors and cell populations involved in skeletal muscle regeneration. JCell Physiol. 2010;224(1):7-16.

26. Brack AS, Rando TA. Tissue-specific stem cells: lessons from the skeletal muscle satellite cell. Cell Stem Cell. 2012;10(5):504-514.

27. Relaix F, Zammit PS. Satellite cells are essential for skeletal muscle regeneration: the cell on the edge returns centre stage. Development. 2012;139(16):2845-2856.

28. Yin $\mathrm{H}$, et al. Satellite cells and the muscle stem cell niche. Physiol Rev. 2013;93(1):23-67.

29. Liu N, et al. A Twist2-dependent progenitor cell contributes to adult skeletal muscle. Nat Cell Biol. 2017;19(3):202-213

30. Egerman MA, Glass DJ. Signaling pathways controlling skeletal muscle mass. Crit Rev Biochem Mol Biol. 2014;49(1):59-68.

31. Ruegg MA, Glass DJ. Molecular mechanisms and treatment options for muscle wasting diseases. Annu Rev Pharmacol Toxicol. 2011;51:373-395.

32. Cohen S, et al. Muscle wasting in disease: molecular mechanisms and promising therapies. Nat Rev Drug Discov. 2015;14(1):58-74.

33. Chamberlain JS, et al. Expression of the murine Duchenne muscular dystrophy gene in muscle and brain. Science. 1988;239(4846):1416-1418.

34. Hoffman EP, et al. Dystrophin: the protein product of the Duchenne muscular dystrophy locus. Cell. 1987;51(6):919-928.

35. Liu Y, et al. Role for the endoplasmic reticulum stress sensor IRE1 $\alpha$ in liver regenerative responses. JHepatol. 2015;62(3):590-598.

36. Xu T, et al. The IRE1 $\alpha-\mathrm{XBP} 1$ pathway regulates metabolic stress-induced compensatory proliferation of pancreatic $\beta$-cells. Cell Res. 2014;24(9):1137-1140.

37. Yan Z, et al. Highly coordinated gene regulation in mouse skeletal muscle regeneration. J Biol Chem. 2003;278(10):8826-8836.

38. Shao M, et al. Hepatic IRE1 $\alpha$ regulates fastinginduced metabolic adaptive programs through the XBP1s-PPAR $\alpha$ axis signalling. Nat Commun . 2014;5:3528.

39. Rebbapragada A, et al. Myostatin signals through a transforming growth factor beta-like signaling pathway to block adipogenesis. Mol Cell Biol. 2003;23(20):7230-7242.

40. Liu L, et al. Isolation of skeletal muscle stem cells by fluorescence-activated cell sorting. Nat Protoc. 2015;10(10):1612-1624.

41. Qiu Y, et al. A crucial role for RACK1 in the regulation of glucose-stimulated IRE1alpha activation in pancreatic beta cells. Sci Signal. 2010;3(106):ra7.

42. Lee SJ. Regulation of muscle mass by myostatin.
Annu Rev Cell Dev Biol. 2004;20:61-86.

43. Trendelenburg AU, et al. Myostatin reduces Akt/ TORC1/p70S6K signaling, inhibiting myoblast differentiation and myotube size. Am J Physiol Cell Physiol. 2009;296(6):C1258-C1270.

44. Rodgers BD, et al. Myostatin stimulates, not inihibits, C2C12 myoblast proliferation. Endocrinology. 2014;155(3):670-675

45. Wang L, et al. Label-free LC-MS/MS proteomics analyses reveal proteomic changes accompanying MSTN KO in C2C12 cells. Biomed Res Int. 2019;2019:7052456.

46. Lee SJ, McPherron AC. Regulation of myostatin activity and muscle growth. Proc Natl Acad Sci US A. 2001;98(16):9306-9311.

47. Thies RS, et al. GDF-8 propeptide binds to GDF-8 and antagonizes biological activity by inhibiting GDF-8 receptor binding. Growth Factors. 2001;18(4):251-259.

48. Hill JJ, et al. The myostatin propeptide and the follistatin-related gene are inhibitory binding proteins of myostatin in normal serum. J Biol Chem. 2002;277(43):40735-40741.

49. Qiao C, et al. Myostatin propeptide gene delivery by adeno-associated virus serotype 8 vectors enhances muscle growth and ameliorates dystrophic phenotypes in mdx mice. Hum Gene Ther. 2008;19(3):241-254.

50. Mouisel E, et al. Myostatin is a key mediator between energy metabolism and endurance capacity of skeletal muscle. Am J Physiol Regul Integr Comp Physiol. 2014;307(4):R444-R454.

51. Cross BC, et al. The molecular basis for selective inhibition of unconventional mRNA splicing by an IRE1-binding small molecule. Proc Natl Acad Sci U S A. 2012;109(15):E869-E878.

52. Volkmann K, et al. Potent and selective inhibitors of the inositol-requiring enzyme 1 endoribonuclease. J Biol Chem. 2011;286(14):12743-12755.

53. Wallace GQ, McNally EM. Mechanisms of muscle degeneration, regeneration, and repair in the muscular dystrophies. Annu Rev Physiol. 2009; 71:37-57.

54. Bentzinger CF, et al. Building muscle: molecular regulation of myogenesis. Cold Spring Harb Perspect Biol. 2012;4(2):a008342.

55. Rodriguez J, et al. Myostatin and the skeletal muscle atrophy and hypertrophy signaling pathways. Cell Mol Life Sci. 2014;71(22):4361-4371.

56. Bogdanovich S, et al. Functional improvement of dystrophic muscle by myostatin blockade. Nature. 2002;420(6914):418-421.

57. Murphy KT, et al. Antibody-directed myostatin inhibition improves diaphragm pathology in young but not adult dystrophic mdx mice. American J Pathol. 2010;176(5):2425-2434.

58. Xiong $\mathrm{G}$, et al. The PERK arm of the unfolded protein response regulates satellite cell-mediated skeletal muscle regeneration. Elife. 2017;6:e22871

59. McCroskery S, et al. Myostatin negatively regulates satellite cell activation and selfrenewal. JCell Biol. 2003;162(6):1135-1147.

60. Rios R, et al. Myostatin regulates cell survival during C2C12 myogenesis. Biochem Biophys Res Commun. 2001;280(2):561-566.

61. Wu Y, et al. Dual role for inositol-requiring enzyme $1 \alpha$ in promoting the development of 
hepatocellular carcinoma during diet-induced obesity in mice. Hepatology. 2018;68(2):533-546.

62. Zhu H, et al. STAT3 regulates self-renewal of adult muscle satellite cells during injury-induced muscle regeneration. Cell Rep.
2016;16(8):2102-2115.

63. Grobet $\mathrm{L}$, et al. A deletion in the bovine myostatin gene causes the double-muscled phenotype in cattle. Nat Genet. 1997;17(1):71-74.

64. Lin J, et al. Myostatin knockout in mice increases myogenesis and decreases adipogenesis. Biochem Biophys Res Commun. 2002;291(3):701-706.

65. McPherron AC, et al. Regulation of skeletal muscle mass in mice by a new TGF-beta superfamily member. Nature. 1997;387(6628):83-90. 